UCLA/04/TEP/46

Columbia/Math/04

2004 November 17

\title{
ASYZYGIES, MODULAR FORMS, AND THE SUPERSTRING MEASURE I *
}

\author{
Eric D'Hoker ${ }^{a}$ and D.H. Phong ${ }^{b}$ \\ ${ }^{a}$ Department of Physics and Astronomy \\ University of California, Los Angeles, CA 90095 \\ ${ }^{b}$ Department of Mathematics \\ Columbia University, New York, NY 10027
}

\begin{abstract}
The goal of this paper and of a subsequent continuation is to find some viable ansatze for the three-loop superstring chiral measure. For this, two alternative formulas are derived for the two-loop superstring chiral measure. Unlike the original formula, both alternates admit modular covariant generalizations to higher genus. One of these two generalizations is analyzed in detail in the present paper, with the analysis of the other left to the next paper of the series.
\end{abstract}

${ }^{*}$ Research supported in part by National Science Foundation grants PHY-01-40151 and DMS-02-45371. 


\section{Introduction}

String perturbation theory is closely related to the mathematical theories of Riemann surfaces and modular forms. In string theory, scattering amplitudes are given perturbatively at loop order $h$ by the moments of a measure on the moduli space of Riemann surfaces of genus $h$. For example, the vacuum-to-vacuum amplitude, or cosmological constant, is given by an expression of the form

$$
\mathcal{A}=\int_{\mathcal{M}_{h}}(\operatorname{det} \operatorname{Im} \Omega)^{-5} \sum_{\delta, \bar{\delta}} c_{\delta, \bar{\delta}} d \mu[\delta](\Omega) \wedge \overline{d \mu[\bar{\delta}](\Omega)}
$$

where $\delta, \bar{\delta}$ denote independent spin structures, $c_{\delta, \bar{\delta}}$ are suitable constant phases, and $d \mu[\delta](\Omega)$ is a holomorphic form of maximal rank $(3 h-3,0)$ on the moduli space of Riemann surfaces of genus $h$. Here we have represented the Riemann surface by its period matrix $\Omega$, after a choice of canonical homology basis. Since the integrand in the above formula should be independent of the homology basis, it follows that $d \mu[\delta](\Omega)$ must transform covariantly under $\operatorname{Sp}(2 h, \mathbf{Z})$ modular transformations.

This correspondence between the superstring measure and modular forms is particularly simple at one-loop, where $d \mu[\delta] \sim \vartheta[\delta](0, \Omega)^{4} / \eta(\Omega)^{12}$, so that $d \mu[\delta](\Omega)$ is essentially a $\vartheta$-constant. No such simple relation is known in general. It has long been hoped that the extensive mathematical literature on modular forms, combined with physical constraints on the superstring measure such as factorization, can help pinpoint the measure. But this hope has remained unfulfilled, perhaps because of the lack of a suitable Ansatz.

In [1, 2, 3, 4] (see also the review [5]), an explicit formula for the two-loop superstring measure was derived from first principles. It is given by

$$
d \mu[\delta]=\frac{\vartheta[\delta]^{4}(0, \Omega) \Xi_{6}[\delta](\Omega)}{\Psi_{10}(\Omega)} \prod_{1 \leq I \leq J \leq 2} d \Omega_{I J}
$$

Here $\Psi_{10}(\Omega)=\prod_{\delta \text { even }} \vartheta[\delta](0, \Omega)^{2}$ is the well-known modular form introduced by Igusa [6. 7], and the key expression $\Xi_{6}[\delta](\Omega)$ is defined by

$$
\Xi_{6}[\delta](\Omega) \equiv \sum_{1 \leq i<j \leq 3}\left\langle\nu_{i} \mid \nu_{j}\right\rangle \prod_{k=4,5,6} \vartheta\left[\nu_{i}+\nu_{j}+\nu_{k}\right]^{4}(0, \Omega),
$$

where we have expressed the even spin structure $\delta$ as the sum of three odd spin structures, say $\delta=\nu_{1}+\nu_{2}+\nu_{3}$, and $\nu_{4}, \nu_{5}, \nu_{6}$ are the remaining odd spin structures. This prescription clearly depends heavily on the fact that the surface has genus 2 .

The main goal of the present paper is to provide two alternative formulas of $\Xi_{6}[\delta](\Omega)$ which are no longer tied specifically to genus 2 . One formula still retains the form (1.3) (so 
that $\Xi_{6}[\delta]$ is still expressed as a linear combination of the 4 th-powers of three $\vartheta$-constants), but the contributing spin structures $\nu_{i}+\nu_{j}+\nu_{4}, \nu_{i}+\nu_{j}+\nu_{5}, \nu_{i}+\nu_{j}+\nu_{6}$ are now characterized as triples $\left\{\delta_{m}, \delta_{n}, \delta_{p}\right\}$ which together with $\delta$ form a totally asyzygous quartet (Theorem 1 ). The other formula is a more radical departure from (1.3), since $\Xi_{6}[\delta]$ is now expressed as a linear combination of the squares of six $\vartheta$-constants. The corresponding sextets of spin structures are characterized by the requirements that they don't contain $\delta$, and can be divided into three pairs of spin structures, the union of any two is a totally asyzygous quartet (Theorem 2).

The new formula in terms of 4 -th powers of $\vartheta$-constants admits immediately a generalization to all genera $h \geq 2$. The generalization to genus 3 of the formula in terms of squares of $\vartheta$-constants is already more delicate. We shall describe it only in the next paper of this series, and devote the rest of this paper to the study of the 4-th powers formula. We show that the generalization of the 4-th powers formula satisfies the same modular transformation laws as in genus 2 (Theorem 3) and the same relation with the basic modular forms $\Psi_{4}(\Omega)^{2}-2^{h} \Psi_{8}(\Omega)$, upon summation over spin structures (Theorem 4). The expression $\Psi_{4}(\Omega)^{2}-2^{h} \Psi_{8}(\Omega)$ is well-known to vanish in genus 1 by the Jacobi identity, and in genus 2 as a consequence of Igusa's theorem on the ring of modular forms. Our numerical simulations indicate very strongly that it also vanishes in genus 3 , and a natural conjecture is that it actually vanishes for all genera. We also determine explicitly the degeneration limits of the 4-th powers formula in genus 3.

As we had stressed earlier, our main motivation for this study is to develop a suitable Ansatz for the superstring measure in higher genus. In the next paper of this series, we shall show, based on the degeneration properties of the two formulas, that it is the formula in terms of squares of $\vartheta$-constants of sextets of spin structures which leads to viable candidates for the three-loop superstring measure. Nevertheless, both formulas exhibit remarkable properties, and it can be hoped that they will both be of interest in the theories of Riemann surfaces and modular forms. 


\section{Alternative formulas for $\Xi_{6}[\delta](\Omega)$}

On a Riemann surface $\Sigma$ of genus $h$, there are $2^{2 h}$ spin structures, i.e., square roots of the canonical bundle of $\Sigma$. Fixing a canonical homology basis, a spin structure $\delta$ can be identified with a half-characteristic $\delta=\left(\delta^{\prime} \mid \delta^{\prime \prime}\right), \delta^{\prime}, \delta^{\prime \prime} \in\left\{0, \frac{1}{2}\right\}^{h}$. The $2^{2 h}$ spin structures can be divided into $2^{h-1}\left(2^{h}+1\right)$ even and $2^{h-1}\left(2^{h}-1\right)$ odd ones, depending on the parity $p(\delta)=\exp \left(4 \pi i \delta^{\prime} \cdot \delta^{\prime \prime}\right)$. The relative signature $\left\langle\delta_{1} \mid \delta_{2}\right\rangle$ of a pair of spin structures $\delta_{1}, \delta_{2}$ is defined by

$$
\left\langle\delta_{1} \mid \delta_{2}\right\rangle=\exp \left\{4 \pi i\left(\delta_{1}^{\prime} \cdot \delta_{2}^{\prime \prime}-\delta_{1}^{\prime \prime} \cdot \delta_{2}^{\prime}\right)\right\}
$$

The relative signature of two spin structures $\delta_{1}, \delta_{2}$ is not invariant under modular transformations (for their precise transformation laws, see Appendix $\S \mathrm{A}$ ). However, for a triplet $\delta, \epsilon, \eta$ of spin structures, we can define the sign $e(\delta, \epsilon, \eta)$ by

$$
e(\delta, \epsilon, \eta) \equiv\langle\delta \mid \epsilon\rangle \cdot\langle\epsilon \mid \eta\rangle \cdot\langle\eta \mid \delta\rangle
$$

which does not depend on the ordering of the triplet, and is invariant under modular transformations. This can be seen from the relation between the parities of a triplet of spin structures and their relative signature, which is a direct consequence of their definitions

$$
p(\delta+\epsilon+\eta)=p(\delta) p(\epsilon) p(\eta) e(\delta, \epsilon, \eta)
$$

In genus $h=2$, there are 10 even spin structures and 6 odd ones. It is convenient to reserve the notation $\delta$ for even spin structures, and denote odd spin structures by $\nu$. Each even spin structure $\delta$ can be written as $\delta=\nu_{1}+\nu_{2}+\nu_{3}$, where the $\nu_{i}$ 's are pairwise distinct odd spin structures. If $\nu_{4}, \nu_{5}, \nu_{6}$ is the complementary set of odd spin structures, then we also have $\delta=\nu_{4}+\nu_{5}+\nu_{6}$, and the mapping $\left\{\nu_{1}, \nu_{2}, \nu_{3}\right\} \rightarrow \delta$ is exactly 2 to 1 . For each even spin structure $\delta$ in genus 2 , say $\delta=\nu_{1}+\nu_{2}+\nu_{3}$, the form $\Xi_{6}[\delta]$ was defined in [1] by the formula (1.3) . In this section, we provide two more formulas for $\Xi_{6}[\delta](\Omega)$. The first one gives a different description of the even spin structures whose $\vartheta$-constants enter the right hand side of (1.3). The second one replaces the product of 3 fourth powers of $\vartheta$-constants by the product of 6 second powers of different $\vartheta$-constants. Both formulas do not depend on the decomposition of even spin structures into odd spin structures in genus 2 and hence allow extensions to arbitrary genus.

Let $\left(\delta_{1}, \cdots, \delta_{N}\right)$ be an unordered $N$-tuplet of $N$ distinct spin structures. We say that the $N$-tuplet is a totally asyzygous $N$-tuplet if $e\left(\delta_{i_{1}}, \delta_{i_{2}}, \delta_{i_{3}}\right)=-1$ for any subset of three distinct spin structures $\delta_{i_{1}}, \delta_{i_{2}}, \delta_{i_{3}}$ in the $N$-tuplet. 
Theorem 1 The expression $\Xi_{6}[\delta](\Omega)$ in (1.3) can be rewritten as

$$
\Xi_{6}[\delta](\Omega)=-\frac{1}{2} \sum_{\delta, \epsilon, \eta, \kappa \text { tot.asyz. }}\langle\delta \mid \epsilon\rangle\langle\delta \mid \eta\rangle\langle\delta \mid \kappa\rangle \cdot \vartheta[\epsilon]^{4}(0, \Omega) \vartheta[\eta]^{4}(0, \Omega) \vartheta[\kappa]^{4}(0, \Omega)
$$

Here the summation is over all triples $\epsilon, \eta, \kappa$ which together with $\delta$ form a totally asyzygous quartet. Each triplet $(\epsilon, \eta, \kappa)$ is counted only once, irrespective of its ordering.

Proof. We have to show that, given an even spin structure $\delta=\nu_{1}+\nu_{2}+\nu_{3}$, the quartets

$$
\left\{\nu_{i}+\nu_{j}+\nu_{k}\right\}_{\{3 \leq k \leq 6\}}
$$

are asygygous for any choice of $i j$ among 12, 13, 23, and that conversely, any totally asyzygous quartet $\{\delta, \epsilon, \eta, \kappa\}$ containing $\delta$ must be of this form (or of the mirror form $\left\{\nu_{i}+\nu_{j}+\nu_{k}\right\}_{\{1 \leq k \leq 4\}}$ if $\delta$ is represented as $\left.\delta=\nu_{4}+\nu_{5}+\nu_{6}\right)$.

We begin by showing that (2.5) is asyzygous. Recall that in genus 2, it follows immediately from the description of even and odd spin structures that $\nu_{i}+\nu_{j}+\nu_{k}$ is even if and only if the odd spin structures $\nu_{i}, \nu_{j}, \nu_{k}$ are pairwise distinct. Thus the spin structures in the quartet in (2.5) are all even. Furthermore, the sum of any distinct three of them is $3\left(\nu_{i}+\nu_{j}\right)+\nu_{k_{1}}+\nu_{k_{2}}+\nu_{k_{3}}=\nu_{i}+\nu_{j}+\nu_{k_{1}}+\nu_{k_{2}}+\nu_{k_{3}}$, and hence equal to the odd spin structure $\nu_{k_{4}}$, where $k_{4}$ is the remaining index in $\{3,4,5,6\}$. It follows now from the relation (2.3) that the triplet $\left\{\nu_{i}+\nu_{j}+\nu_{k}\right\}_{\left\{k=k_{1}, k_{2}, k_{3}\right\}}$ is asyzygous, and hence the quartets (2.5) are totally asyzygous.

We show now the converse. Since all the spin structures in $\{\delta, \epsilon, \eta, \kappa\}$ are assumed to be even, the totally asyzygous condition is equivalent, in view of the relation (2.3) to the condition that the sum of any three distinct spin structures in the quartet be odd. We can easily work this out for $\delta=\nu_{1}+\nu_{2}+\nu_{3}$. Recall that each even spin structure admits two representations, as the sum of a set of three distinct odd spin structures $\nu^{\prime}+\nu^{\prime \prime}+\nu^{\prime \prime \prime}$, or as the complementary sum. Since the spin structures $\epsilon, \eta, \kappa$ are different from $\delta$, we may assume that, for each of them, at least one of the three spin structures $\nu^{\prime}, \nu^{\prime \prime}, \nu^{\prime \prime \prime}$ is among $\nu_{1}, \nu_{2}, \nu_{3}$. By taking the complementary representation if necessary, we may assume that at least two of them are in this set, and in fact exactly two, since they are distinct from $\delta$. Thus, after some suitable renaming, the spin structures $\epsilon, \eta, \kappa$ must fall into one of the following three possibilities
(1) $\left\{\begin{array}{l}\epsilon=\nu_{1}+\nu_{2}+\nu_{j} \\ \eta=\nu_{1}+\nu_{3}+\nu_{k} \\ \kappa=\nu_{2}+\nu_{3}+\nu_{l}\end{array}\right.$
(2) $\left\{\begin{array}{l}\epsilon=\nu_{1}+\nu_{2}+\nu_{j} \\ \eta=\nu_{1}+\nu_{2}+\nu_{k} \\ \kappa=\nu_{1}+\nu_{3}+\nu_{l}\end{array}\right.$
(3) $\left\{\begin{array}{l}\epsilon=\nu_{1}+\nu_{2}+\nu_{j} \\ \eta=\nu_{1}+\nu_{2}+\nu_{k} \\ \kappa=\nu_{1}+\nu_{2}+\nu_{l}\end{array}\right.$

with $\nu_{j}, \nu_{k}, \nu_{l} \in\{4,5,6\}$. 
The case (3) gives a quartet of type (2.5), as claimed. To deal with the cases (1) and (2), we again exploit the fact that the sum of the spin structures in an asygygous triplet of even spin structures must be odd. Applied to a triplet of the form $\left\{\delta=\nu_{1}+\nu_{2}+\nu_{3}, \nu_{1}+\right.$ $\left.\nu_{2}+\nu_{j}, \nu_{1}+\nu_{3}+\nu_{k}\right\}$, it implies that $\nu_{3}+\nu_{j}+\nu_{k}$ must be odd. But this implies that $\nu_{j}=\nu_{k}$ (since they are both different from $\nu_{3}$ ). Turning more specifically to the case (1), we deduce that the spin structures $\nu_{j}, \nu_{k}, \nu_{l}$ must all be the same in this case. Going to the complementary representation for $\{\delta, \epsilon, \eta, \kappa\}$, we obtain a quartet of the form (2.5), with $i j$ given by one of the pairs 45,46 , or 56 . Finally, for case (2), we note that the previous observation implies that $\nu_{k}=\nu_{l}$. But then we have $\epsilon+\eta+\kappa=\nu_{1}+\nu_{3}+\nu_{j}$, which is even, contradicting the fact that the triplet $\epsilon, \eta, \kappa$ is asyzygous.

It remains to re-express the sign factor $\left\langle\nu_{i}, \nu_{j}\right\rangle$ in (1.3) in the form of indicated in (2.4). For this, consider an asyzygous quartet $\{\delta, \epsilon, \eta, \kappa\}$ written under the form corresponding to case (3) above. Then $\langle\delta \mid \epsilon\rangle\langle\delta \mid \eta\rangle\langle\delta \mid \kappa\rangle=\langle\delta \mid \epsilon+\eta+\kappa\rangle=\left\langle\delta \mid \nu_{3}\right\rangle=\left\langle\nu_{1} \mid \nu_{3}\right\rangle\left\langle\nu_{2} \mid \nu_{3}\right\rangle$. Since the triplet $\left\{\nu_{1}, \nu_{2}, \nu_{3}\right\}$ is asyzygous, this is equal to $-\left\langle\nu_{1} \mid \nu_{2}\right\rangle$. Q.E.D.

Theorem 2 The form $\Xi_{6}[\delta](0, \Omega)$ can also be expressed as

$$
\Xi_{6}[\delta](\Omega)=\frac{1}{2} \sum_{\left\{\delta_{i}\right\}} \epsilon\left(\delta ;\left\{\delta_{i}\right\}\right) \prod_{j=1}^{6} \vartheta\left[\delta_{i_{j}}\right](0, \Omega)^{2},
$$

where the sum runs over all sextets $\left\{\delta_{i_{1}}, \cdots, \delta_{i_{6}}\right\}$ of even spin structures which are $\delta$ admissible in the following sense: (1) $\delta \notin\left\{\delta_{i_{j}}\right\}$; and (2) the sextet can be partitioned into 3 disjoint pairs $\left\{\delta_{i_{1}}, \delta_{i_{2}}\right\},\left\{\delta_{i_{3}}, \delta_{i_{4}}\right\},\left\{\delta_{i_{5}}, \delta_{i_{6}}\right\}$, the union of any two pairs forming a totally asyzygous quartet.

The modular group $\operatorname{Sp}\left(4, \mathbf{Z}_{2}\right)$ acts transitively on the space of 7 -tuplets $\left\{\delta ; \delta_{i_{1}}, \cdots, \delta_{i_{6}}\right\}$ satisfying the condition that $\left\{\delta_{i_{1}}, \cdots, \delta_{i_{6}}\right\}$ id $\delta$-admissible. The expressions $\epsilon\left(\delta ; \delta_{i_{1}}, \cdots, \delta_{i_{6}}\right) \in$ $\{ \pm 1\}$ are phases satisfying the following modular transformation law

$$
\epsilon\left(M \delta ;\left\{M \delta_{i_{j}}\right\}\right) \epsilon(\delta, M)^{4}=\epsilon\left(\delta ;\left\{\delta_{i_{j}}\right\}\right) \prod_{k=1}^{6} \epsilon\left(\delta_{i_{k}}, M\right)^{2}
$$

where $\epsilon(\delta, M)$ is the 8-th root of unity entering transformation laws for $\vartheta$-constants. The phases $\epsilon\left(\delta ; \delta_{i_{1}}, \cdots, \delta_{i_{6}}\right)$ are completely determined by one another by this transformation law. The global relative sign in (2.7) is determined by the choice $\epsilon\left(\delta_{7} ;\left\{\delta_{3}, \delta_{4}, \delta_{5}, \delta_{6}, \delta_{9}, \delta_{0}\right\}\right)=$ -1 , in the indexing of even spin structures defined in Appendix $\S B$. Their explicit form will be given below, in (2.22) and (2.23). 
Proof. The main tool in the proof is the Riemann relations for $\vartheta$-functions. Riemann relations are usually applied to 4th-powers of $\vartheta$-constants, but they apply equally well to 2nd powers, as we show now. Recall that the Riemann relations are given by [4], §2.4

$$
\sum_{\lambda}\langle\kappa \mid \lambda\rangle \vartheta[\lambda]\left(\zeta_{1}\right) \vartheta[\lambda]\left(\zeta_{2}\right) \vartheta[\lambda]\left(\zeta_{3}\right) \vartheta[\lambda]\left(\zeta_{4}\right)=4 \prod_{i=1}^{4} \vartheta[\kappa]\left(\zeta_{1}^{\prime}\right)
$$

where $\zeta^{\prime}$ is expressed as linear combinations of the $\zeta$. The case of interest here is for $\zeta_{1}=\zeta_{2}=\varepsilon$ and $\zeta_{3}=\zeta_{4}=0$, where $\varepsilon \neq 0$ is a "twist" half-characteristic. Thus, the relations reduce to $\zeta_{1}^{\prime}=\zeta_{2}^{\prime}=\varepsilon$ and $\zeta_{3}^{\prime}=\zeta_{4}^{\prime}=0$. Restricting attention to $\kappa=\nu$ odd, we are left with

$$
0=\sum_{\delta}\langle\delta \mid \nu\rangle \vartheta[\delta](\varepsilon)^{2} \vartheta[\delta](0)^{2}=\sum_{\delta} e^{-4 \pi i \varepsilon^{\prime} \delta^{\prime \prime}}\langle\delta \mid \nu\rangle \vartheta[\delta+\varepsilon](0)^{2} \vartheta[\delta](0)^{2}
$$

This sum receives non-trivial contributions only from terms with both $\delta$ and $\delta+\varepsilon$ even. Any of the 15 twists may be expressed (see for example [8]) as the sum of two odd spin structures, $\varepsilon=\nu_{a}+\nu_{b}$ for $a \neq b$, all such spin structures may be parametrized by

$$
\delta=\nu_{a}+\nu_{j}+\nu_{k}, \quad \delta+\varepsilon=\nu_{b}+\nu_{j}+\nu_{k} \quad j, k \notin\{a, b\}
$$

Of the 6 possible odd spin structures, 4 yield trivially 0 . The two (equivalent) non-trivial cases are for $\nu=\nu_{a}$ and $\nu=\nu_{b}$. Choosing the first, the Riemann identity may now be recast in the following form,

$$
0=\sum_{\substack{j \neq k \\ j, k \neq a, b}} \varphi(a b \mid j k) \vartheta\left[\nu_{a}+\nu_{j}+\nu_{k}\right](0)^{2} \vartheta\left[\nu_{b}+\nu_{j}+\nu_{k}\right](0)^{2}
$$

where

$$
\varphi(a b \mid j k)=\varphi(b a \mid j k)=\exp 4 \pi i\left\{\nu_{a}^{\prime \prime}\left(\nu_{j}^{\prime}+\nu_{k}^{\prime}\right)+\nu_{b}^{\prime}\left(\nu_{j}^{\prime \prime}+\nu_{k}^{\prime \prime}\right)\right\}
$$

Here, the symmetry in $a \leftrightarrow b$ is readily shown using the fact that $j \neq k$ and that $a, b \notin$ $\{j, k\}$. This form of Riemann identities is closely related to Riemann identities on Prym varieties, which appeared in the study of the cosmological constant in certain orbifold models with broken supersymmetry [8, 11].

We can return now to the proof of Theorem 2 proper. The property (1) of $\delta$-admissible sextets described in Theorem 2 is clearly invariant under modular transformations. Both properties (1) and (2) are invariant under the subgroup of modular transformations which leave the spin structure $\delta$ unchanged. The transitivity of this subgroup can be read off from the tables of sextets and modular transformations in Appendix $\S \mathrm{B}$. The transformation 
law for the phases $\epsilon\left(\delta ;\left\{\delta_{i_{j}}\right\}\right)$ implies that both sides of the equation (2.7) transform in the same way under modular transformations. Thus it suffices to prove the theorem for some fixed even spin structure, say $\delta=\nu_{1}+\nu_{2}+\nu_{3}$. This is $\delta_{7}$ in the notation of Appendix $\S \mathrm{B}$. In this case, the sextets which satisfy the properties (1) and (2) are given by (see table in Appendix $\S \mathrm{C}$ )

$$
\{345960\}, \quad\{192638\}, \quad\{152048\}, \quad\{125690\}, \quad\{134589\}, \quad\{234680\} .
$$

where we have abbreviated the sextet $\left\{\delta_{i_{1}}, \cdots, \delta_{i_{6}}\right\}$ by $\left\{i_{1} \cdots i_{6}\right\}$.

We rewrite now $\Xi_{6}\left[\nu_{1}+\nu_{2}+\nu_{3}\right]$ using the preceding version of Riemann identities with squares of $\vartheta$-constants. Using the shorthand $(i j k)=\vartheta\left[\nu_{i}+\nu_{j}+\nu_{k}\right](0)^{2}$, we have the following Riemann relations needed to recast $\Xi_{6}\left[\nu_{1}+\nu_{2}+\nu_{3}\right]$,

$$
\begin{aligned}
& \varphi(45 \mid 12)(124)(125)=-\varphi(45 \mid 13)(134)(135)-\varphi(45 \mid 23)(234)(235) \\
& \varphi(56 \mid 12)(125)(126)=-\varphi(56 \mid 13)(135)(136)-\varphi(56 \mid 23)(235)(236) \\
& \varphi(64 \mid 12)(124)(126)=-\varphi(64 \mid 13)(134)(136)-\varphi(64 \mid 23)(234)(236)
\end{aligned}
$$

Multiplying the three left hand sides together and the three right hand sides together, and using the identity

$$
\varphi(45 \mid i j) \varphi(56 \mid i j) \varphi(64 \mid i j)=-\left\langle\nu_{i} \mid \nu_{j}\right\rangle
$$

we readily see that

$$
\begin{aligned}
\Xi_{6}\left[\nu_{1}+\nu_{2}+\nu_{3}\right]= & -\varphi(45 \mid 13) \varphi(56 \mid 13) \varphi(64 \mid 23)(134)(135)^{2}(136)(234)(236) \\
& -\varphi(45 \mid 13) \varphi(56 \mid 23) \varphi(64 \mid 13)(134)^{2}(135)(136)(235)(236) \\
& -\varphi(45 \mid 23) \varphi(56 \mid 13) \varphi(64 \mid 13)(134)(135)(136)^{2}(234)(235) \\
& -\varphi(45 \mid 23) \varphi(56 \mid 13) \varphi(64 \mid 23)(135)(136)(234)^{2}(235)(236) \\
& -\varphi(45 \mid 23) \varphi(56 \mid 23) \varphi(64 \mid 13)(134)(136)(234)(235)^{2}(236) \\
& -\varphi(45 \mid 13) \varphi(56 \mid 23) \varphi(64 \mid 23)(134)(135)(234)(235)(236)^{2}(2.17)
\end{aligned}
$$

Combining lines 1 and 4, lines 2 and 6, and lines 3 and 5 using the same Riemann identities (2.15), we get

$$
\begin{aligned}
\Xi_{6}\left[\nu_{1}+\nu_{2}+\nu_{3}\right]= & -c_{A}(124)(125)(135)(136)(234)(236) \\
& -c_{B}(125)(126)(134)(136)(234)(235) \\
& -c_{C}(124)(126)(134)(135)(235)(236)
\end{aligned}
$$


The signs are easily simplified, and we get

$$
\begin{aligned}
& c_{A}=\varphi(45 \mid 12) \varphi(56 \mid 13) \varphi(64 \mid 23)=\exp 4 \pi i\left\{\nu_{1}^{\prime} \nu_{6}^{\prime \prime}+\nu_{2}^{\prime} \nu_{5}^{\prime \prime}+\nu_{3}^{\prime} \nu_{4}^{\prime \prime}+\nu_{4}^{\prime} \nu_{1}^{\prime \prime}+\nu_{5}^{\prime} \nu_{3}^{\prime \prime}+\nu_{6}^{\prime} \nu_{2}^{\prime \prime}\right\} \\
& c_{B}=\varphi(45 \mid 23) \varphi(56 \mid 12) \varphi(64 \mid 13)=\exp 4 \pi i\left\{\nu_{2}^{\prime} \nu_{6}^{\prime \prime}+\nu_{3}^{\prime} \nu_{5}^{\prime \prime}+\nu_{1}^{\prime} \nu_{4}^{\prime \prime}+\nu_{4}^{\prime} \nu_{2}^{\prime \prime}+\nu_{5}^{\prime} \nu_{1}^{\prime \prime}+\nu_{6}^{\prime} \nu_{3}^{\prime \prime}\right\} \\
& c_{C}=\varphi(45 \mid 12) \varphi(56 \mid 13) \varphi(64 \mid 23)=\exp 4 \pi i\left\{\nu_{3}^{\prime} \nu_{6}^{\prime \prime}+\nu_{1}^{\prime} \nu_{5}^{\prime \prime}+\nu_{2}^{\prime} \nu_{4}^{\prime \prime}+\nu_{4}^{\prime} \nu_{3}^{\prime \prime}+\nu_{5}^{\prime} \nu_{2}^{\prime \prime}+\nu_{6}^{\prime} \nu_{1}^{\prime \prime}\right\}
\end{aligned}
$$

To re-express the above result in terms of even spin structures, we need to choose a basis for the even spin structures in terms of the odd ones. We choose the same basis as was used in [4], but the actual values of the odd spin structures $\nu_{a}$ are left arbitrary. Modular transformations simply permute the odd spin structures. The basis may be expressed as follows (up to complete periods),

$$
\begin{array}{ll}
\delta_{1}=\nu_{1}+\nu_{4}+\nu_{6}=\nu_{2}+\nu_{3}+\nu_{5} & \delta_{6}=\nu_{1}+\nu_{5}+\nu_{6}=\nu_{2}+\nu_{3}+\nu_{4} \\
\delta_{2}=\nu_{1}+\nu_{2}+\nu_{6}=\nu_{3}+\nu_{4}+\nu_{5} & \delta_{7}=\nu_{1}+\nu_{2}+\nu_{3}=\nu_{4}+\nu_{5}+\nu_{6} \\
\delta_{3}=\nu_{1}+\nu_{2}+\nu_{5}=\nu_{3}+\nu_{4}+\nu_{6} & \delta_{8}=\nu_{1}+\nu_{3}+\nu_{4}=\nu_{2}+\nu_{5}+\nu_{6} \\
\delta_{4}=\nu_{1}+\nu_{4}+\nu_{5}=\nu_{2}+\nu_{3}+\nu_{6} & \delta_{9}=\nu_{1}+\nu_{3}+\nu_{6}=\nu_{2}+\nu_{4}+\nu_{5} \\
\delta_{5}=\nu_{1}+\nu_{2}+\nu_{4}=\nu_{3}+\nu_{5}+\nu_{6} & \delta_{0}=\nu_{1}+\nu_{3}+\nu_{5}=\nu_{2}+\nu_{4}+\nu_{6}
\end{array}
$$

In total, there are 15 totally asyzygous quartets, 9 of which do not contain a given spin structure. The 9 asyzygous quartets which do not contain, say $\delta_{7}$, may be grouped in terms of two groups of three $\delta_{7}$-admissible sextets, given as follows,

$$
\begin{array}{ll}
A=\left[\begin{array}{lll}
34 & 59 & 60
\end{array}\right] & A^{\prime}=\left[\begin{array}{lll}
18 & 39 & 45
\end{array}\right] \\
B=\left[\begin{array}{lll}
19 & 26 & 38
\end{array}\right] & B^{\prime}=\left[\begin{array}{lll}
28 & 36 & 40
\end{array}\right] \\
C=\left[\begin{array}{lll}
15 & 20 & 48
\end{array}\right] & C^{\prime}=\left[\begin{array}{lll}
12 & 50 & 69
\end{array}\right]
\end{array}
$$

The structure of the asyzygous quartets allows us to recast odd spin structures in terms of asyzygous triplets of even ones, for each group, as follows in Table 1 below.

Re-expressing the signs $c_{A}, c_{B}, c_{C}$, in terms of even spin structures, we obtain,

$$
\begin{aligned}
\Xi_{6}\left[\delta_{7}\right]= & -\psi\left(\delta_{4}, \delta_{5}, \delta_{0}\right) \psi\left(\delta_{3}, \delta_{9}, \delta_{6}\right) \vartheta\left[\delta_{3}\right]^{2} \vartheta\left[\delta_{4}\right]^{2} \vartheta\left[\delta_{5}\right]^{2} \vartheta\left[\delta_{6}\right]^{2} \vartheta\left[\delta_{9}\right]^{2} \vartheta\left[\delta_{0}\right]^{2} \\
& -\psi\left(\delta_{3}, \delta_{9}, \delta_{6}\right) \psi\left(\delta_{1}, \delta_{2}, \delta_{8}\right) \vartheta\left[\delta_{1}\right]^{2} \vartheta\left[\delta_{2}\right]^{2} \vartheta\left[\delta_{3}\right]^{2} \vartheta\left[\delta_{6}\right]^{2} \vartheta\left[\delta_{8}\right]^{2} \vartheta\left[\delta_{9}\right]^{2} \\
& -\psi\left(\delta_{1}, \delta_{2}, \delta_{8}\right) \psi\left(\delta_{4}, \delta_{5}, \delta_{0}\right) \vartheta\left[\delta_{1}\right]^{2} \vartheta\left[\delta_{2}\right]^{2} \vartheta\left[\delta_{4}\right]^{2} \vartheta\left[\delta_{5}\right]^{2} \vartheta\left[\delta_{8}\right]^{2} \vartheta\left[\delta_{0}\right]^{2}
\end{aligned}
$$

where the following combination enters,

$$
\psi\left(\delta_{i}, \delta_{j}, \delta_{k}\right) \equiv \exp 4 \pi i\left\{\delta_{i}^{\prime} \delta_{j}^{\prime \prime}+\delta_{j}^{\prime} \delta_{k}^{\prime \prime}+\delta_{k}^{\prime} \delta_{i}^{\prime \prime}\right\}
$$




\begin{tabular}{|c||c|c|c||c|c|c|}
\hline$\nu$ & $\mathrm{A}$ & $\mathrm{B}$ & $\mathrm{C}$ & $\mathrm{A}^{\prime}$ & $\mathrm{B}^{\prime}$ & $\mathrm{C}^{\prime}$ \\
\hline \hline$\nu_{1}$ & $\delta_{3}+\delta_{4}+\delta_{5}$ & $\delta_{1}+\delta_{8}+\delta_{9}$ & $\delta_{4}+\delta_{8}+\delta_{0}$ & $\delta_{1}+\delta_{8}+\delta_{9}$ & $\delta_{2}+\delta_{3}+\delta_{6}$ & $\delta_{1}+\delta_{2}+\delta_{5}$ \\
\hline$\nu_{2}$ & $\delta_{3}+\delta_{5}+\delta_{9}$ & $\delta_{1}+\delta_{6}+\delta_{9}$ & $\delta_{1}+\delta_{4}+\delta_{8}$ & $\delta_{1}+\delta_{4}+\delta_{8}$ & $\delta_{2}+\delta_{3}+\delta_{8}$ & $\delta_{1}+\delta_{6}+\delta_{9}$ \\
\hline$\nu_{3}$ & $\delta_{3}+\delta_{4}+\delta_{6}$ & $\delta_{1}+\delta_{2}+\delta_{6}$ & $\delta_{1}+\delta_{4}+\delta_{5}$ & $\delta_{1}+\delta_{4}+\delta_{5}$ & $\delta_{2}+\delta_{8}+\delta_{0}$ & $\delta_{1}+\delta_{2}+\delta_{6}$ \\
\hline$\nu_{4}$ & $\delta_{3}+\delta_{6}+\delta_{0}$ & $\delta_{1}+\delta_{3}+\delta_{8}$ & $\delta_{2}+\delta_{4}+\delta_{8}$ & $\delta_{1}+\delta_{3}+\delta_{8}$ & $\delta_{2}+\delta_{4}+\delta_{8}$ & $\delta_{1}+\delta_{5}+\delta_{0}$ \\
\hline$\nu_{5}$ & $\delta_{3}+\delta_{4}+\delta_{9}$ & $\delta_{1}+\delta_{2}+\delta_{9}$ & $\delta_{2}+\delta_{4}+\delta_{0}$ & $\delta_{1}+\delta_{5}+\delta_{8}$ & $\delta_{2}+\delta_{4}+\delta_{0}$ & $\delta_{1}+\delta_{2}+\delta_{9}$ \\
\hline$\nu_{6}$ & $\delta_{3}+\delta_{4}+\delta_{0}$ & $\delta_{1}+\delta_{3}+\delta_{9}$ & $\delta_{4}+\delta_{5}+\delta_{8}$ & $\delta_{1}+\delta_{3}+\delta_{9}$ & $\delta_{2}+\delta_{6}+\delta_{8}$ & $\delta_{1}+\delta_{2}+\delta_{0}$ \\
\hline
\end{tabular}

Table 1: Decomposition of odd into even within a $\delta_{7}$-admissible sextet

and our final expression for $\Xi_{6}\left[\delta_{7}\right]$. An alternative formula is obtained by replacing the partitioning $A, B, C$ by $A^{\prime}, B^{\prime}, C^{\prime}$. Averaging over the two formulas gives the desired formula (2.7). Note that it is the full set of sextets in (2.14) which is an orbit under the group of modular transformations fixing $\delta$. Note also that the above expression in terms of even spin structures is uniquely defined by the partitioning. This may be established using the symmetries of the function $\psi$. Computing the effect of a permutation, we find

$$
\psi\left(\delta_{i}, \delta_{j}, \delta_{k}\right) \psi\left(\delta_{j}, \delta_{i}, \delta_{k}\right)=\left\langle\delta_{i} \mid \delta_{j}\right\rangle\left\langle\delta_{j} \mid \delta_{k}\right\rangle\left\langle\delta_{k} \mid \delta_{i}\right\rangle=e\left(\delta_{i}, \delta_{j}, \delta_{k}\right)
$$

Next, by inspection, we see that the triplets $\left(\delta_{i}, \delta_{j}, \delta_{k}\right)$ on which $\psi$ is evaluated in each partition, namely $\left(\delta_{4}, \delta_{5}, \delta_{0}\right),\left(\delta_{3}, \delta_{9}, \delta_{6}\right)$, and $\left(\delta_{1}, \delta_{2}, \delta_{8}\right)$, are all syzygous, so that $\psi\left(\delta_{i}, \delta_{j}, \delta_{k}\right)$ is totally symmetric in its three arguments. Q.E.D.

\section{Remarks}

- We note that, unlike in the expression for $\Xi_{6}[\delta]$ in terms of 4 th-powers of $\vartheta$-constants, the phases $\epsilon\left(\delta ;\left\{\delta_{i}\right\}\right)$ in the expression (2.7) cannot be expressed in terms of simple modular invariant quantities such as the relative signature of two spin structures. This will account for some important subtleties when we discuss the generalization of expressions such as (2.7) to higher genus in [10].

- Another proof of Theorem 2 can be obtained, using the hyperelliptic representation $s^{2}=\prod_{i=1}^{6}\left(x-p_{i}\right)$ of genus 2 Riemann surfaces. If we view the odd spin structures $\nu_{i}$ as corresponding to the branch points $p_{i}$, then the spin structure $\delta=\delta_{7}$ corresponds to the partition of the 6 branch points into two sets $A=\left\{p_{1}, p_{2}, p_{3}\right\}$ and $B=\left\{p_{4}, p_{5}, p_{6}\right\}$. We can then establish the following formula

$$
\frac{\Xi_{6}[\delta]}{\vartheta[\delta]^{2} \Psi_{10}^{1 / 2}}=\frac{\sum_{\sigma}\left(p_{1}-p_{\sigma(4)}\right)\left(p_{2}-p_{\sigma(5)}\right)\left(p_{3}-p_{\sigma(6)}\right)}{\sqrt{\prod_{i \neq j \in A}\left(p_{i}-p_{j}\right) \prod_{i \neq j \in B}\left(p_{i}-p_{j}\right)}}
$$


where $\sigma$ runs over the 3 cyclic permutations of $\{4,5,6\}$. Expressing the right hand side in terms of $\vartheta$-constants gives again (2.18), up to an overall sign, which cannot be determined from the hyperelliptic representation.

- We stress that the full orbit under the subgroup of modular transformations leaving $\delta_{7}$ unchanged requires all 6 sextets listed in (2.14). However, the fact that this orbit can be broken into two groups, each already giving essentially $\Xi_{6}[\delta]$, plays an important role in the construction in [10] of modular forms using pairs of sextets. 


\section{A modular covariant form $\Xi_{6}^{\#}[\delta](\Omega)$ in any genus}

In the previous section, we have seen that the modular covariant form $\Xi_{6}[\delta](\Omega)$ admits an expression in terms of totally asyzygous quartets of even spin structures. This notion makes sense for all genera, and thus we can define an extension to all genera of $\Xi_{6}[\delta](\Omega)$, denoted by $\Xi_{6}^{\#}[\delta](\Omega)$, by the right hand side of (2.4 $)$. The main goal of the present section is to show that $\Xi_{6}^{\#}[\delta](\Omega)$ satisfies modular transformation laws and identities similar to those of $\Xi_{6}[\delta](\Omega)$ in genus 2. But before we do so, we wish to point out that the notion of totally asyzygous odd spin structures could have been used as well.

Let $\left\{\delta_{1}, \delta_{2}, \delta_{3}, \delta_{4}\right\}$ be any quartet of spin structures, and define another quartet of spin structures $\left\{\nu_{1}, \nu_{2}, \nu_{3}, \nu_{4}\right\}$ by

$$
\nu_{i}=\sum_{j \neq i} \delta_{j}, \quad 1 \leq i \leq 4 .
$$

The two quartets determine each other uniquely, since the above equations imply that $\delta_{j}=\sum_{i \neq j} \nu_{i}$. We claim now that $\left\{\delta_{1}, \delta_{2}, \delta_{3}, \delta_{4}\right\}$ is an asyzygous quartet of even spin structures if and only if $\left\{\nu_{1}, \nu_{2}, \nu_{3}, \nu_{4}\right\}$ is an asyzygous quartet of odd spin structures. Indeed, assume that $\left\{\delta_{1}, \delta_{2}, \delta_{3}, \delta_{4}\right\}$ is an asyzygous quartet of even spin structures, and consider say $p\left(\nu_{1}\right)$. Then $p\left(\nu_{1}\right)=p\left(\delta_{2}\right) p\left(\delta_{3}\right) p\left(\delta_{4}\right) e\left(\delta_{2}, \delta_{3}, \delta_{4}\right)=-1$ which shows that $\nu_{1}$, and hence any $\nu_{i}$, is odd. Reversing the roles of $\delta_{i}$ and $\nu_{i}$ in the same formula shows that $p\left(\delta_{1}\right)=p\left(\nu_{2}\right) p\left(\nu_{3}\right) p\left(\nu_{4}\right) e\left(\nu_{2}, \nu_{3}, \nu_{4}\right)$, which implies that $e\left(\nu_{2}, \nu_{3}, \nu_{4}\right)=-1$, since the $\delta_{i}$ 's are even, and the $\nu_{i}$ 's are now known to be odd. Thus the quartet $\left\{\nu_{1}, \nu_{2}, \nu_{3}, \nu_{4}\right\}$ is asyzygous. The same argument can clearly be reversed to show that $\left\{\delta_{1}, \delta_{2}, \delta_{3}, \delta_{4}\right\}$ is an asyzygous quartet of even spin structures if $\left\{\nu_{1}, \nu_{2}, \nu_{3}, \nu_{4}\right\}$ is an asyzygous quartet of odd spin structures, completing the proof of our claim.

In view of the above exact correspondence between asyzygous quartets of even and odd spin structures, we obtain immediately the following equivalent characterization of $\Xi_{6}^{\#}[\delta](\Omega)$ valid in any genus

$$
\Xi_{6}^{\#}[\delta]=\frac{1}{6} \sum_{\left[\nu_{1}, \nu_{2}, \nu_{3}, \nu_{4}\right]} \theta_{\left[\nu_{1}, \nu_{2}, \nu_{3}, \nu_{4}\right]}[\delta]\left\langle\delta \mid \delta_{\sigma(1)}\right\rangle\left\langle\delta \mid \delta_{\sigma(2)}\right\rangle\left\langle\delta \mid \delta_{\sigma(3)}\right\rangle \vartheta\left[\delta_{\sigma(1)}\right]^{4} \vartheta\left[\delta_{\sigma(2)}\right]^{4} \vartheta\left[\delta_{\sigma(3)}\right]^{4}
$$

Here the summation is over all totally asyzygous quartets $\left[\nu_{1}, \nu_{2}, \nu_{3}, \nu_{4}\right]$ of odd spin structures. The indicator function is defined by

$$
\theta_{\left[\nu_{1}, \nu_{2}, \nu_{3}, \nu_{4}\right]}[\delta] \equiv\left\{\begin{array}{lll}
1 & \text { iff } & \delta=\nu_{\sigma(1)}+\nu_{\sigma(2)}+\nu_{\sigma(3)} \\
0 & \text { iff } & \delta \neq \nu_{\sigma(1)}+\nu_{\sigma(2)}+\nu_{\sigma(3)}
\end{array}\right.
$$

where $\sigma$ is any permutation of $1,2,3,4$.

We turn now to the modular properties of $\Xi_{6}^{\#}[\delta](\Omega)$ : 
Theorem 3 Under modular transformations $M$, the expression $\Xi_{6}^{\#}[\delta](\Omega)$ transforms as

$$
\Xi_{6}^{\#}[\tilde{\delta}](\tilde{\Omega})=\epsilon(\delta, M)^{4} \operatorname{det}(C \Omega+D)^{6} \Xi_{6}^{\#}[\delta](\Omega)
$$

where $\tilde{\epsilon}=M \epsilon$ and $\epsilon(\delta, M)$ is precisely the same 8-th root of unity occurring in the modular transformation for $\vartheta[\delta](0, \Omega)$.

Proof. We establish (3.4) for general modular transformations by establishing it for the generators of $S p(2 h, \mathbf{Z})$. A set of generators is given by the matrices $M_{A}, S$, and $M_{B}$ listed in Appendix $\S$ A. Modular transformations map totally asyzygous quartets into totally asyzygous quartets. Now the signature on pairs is invariant under $M_{A}$ and $S$, and the factors $\epsilon\left(\delta, M_{A}\right)$ and $\epsilon(\delta, S)$ are essentially trivial in this case, so we need only consider the case of $M_{B}$ generators. We proceed as follows,

$$
\Xi_{6}^{\#}[\tilde{\delta}](\tilde{\Omega})=-\frac{1}{2} \sum_{[\tilde{\delta}, \tilde{\epsilon}, \tilde{\eta}, \tilde{\kappa}]}\langle\tilde{\delta} \mid \tilde{\epsilon}\rangle\langle\tilde{\delta} \mid \tilde{\eta}\rangle\langle\tilde{\delta} \mid \tilde{\kappa}\rangle \cdot \vartheta[\tilde{\epsilon}]^{4}(0, \tilde{\Omega}) \vartheta[\tilde{\eta}]^{4}(0, \tilde{\Omega}) \vartheta[\tilde{\kappa}]^{4}(0, \tilde{\Omega})
$$

Next, we cast each spin structure as the transformation of its pre-image, and use the $\vartheta$-constant transformation laws,

$$
\begin{gathered}
\Xi_{6}^{\#}[\tilde{\delta}](\tilde{\Omega})=-\frac{1}{2} \sum_{[\delta, \epsilon, \eta, \kappa]}\langle\tilde{\delta} \mid \tilde{\epsilon}\rangle\langle\tilde{\delta} \mid \tilde{\eta}\rangle\langle\tilde{\delta} \mid \tilde{\kappa}\rangle \epsilon^{4}\left(\epsilon, M_{B}\right) \epsilon^{4}\left(\eta, M_{B}\right) \epsilon^{4}\left(\kappa, M_{B}\right) \\
\times \vartheta[\epsilon]^{4}(0, \Omega) \vartheta[\eta]^{4}(0, \Omega) \vartheta[\kappa]^{4}(0, \Omega)
\end{gathered}
$$

The signature factors worked out to be

$$
\begin{array}{r}
\langle\tilde{\delta} \mid \tilde{\epsilon}\rangle\langle\tilde{\delta} \mid \tilde{\eta}\rangle\langle\tilde{\delta} \mid \tilde{\kappa}\rangle=\langle\delta \mid \epsilon\rangle\langle\delta \mid \eta\rangle\langle\delta \mid \kappa\rangle \exp \left\{2 \pi i \sum_{i=1}^{h}\left(3 \delta_{i}^{\prime}-\epsilon_{i}^{\prime}-\eta_{i}^{\prime}-\kappa_{i}^{\prime}\right) B_{i}\right\} \\
\epsilon^{4}\left(\epsilon, M_{B}\right) \epsilon^{4}\left(\eta, M_{B}\right) \epsilon^{4}\left(\kappa, M_{B}\right)=\exp \left\{-4 \pi i \sum_{i=1}^{h}\left(\left(\epsilon_{i}^{\prime}\right)^{2}+\left(\eta_{i}^{\prime}\right)^{2}+\left(\kappa_{i}^{\prime}\right)^{2}\right) B_{i}\right\}
\end{array}
$$

Assembling both factors, and using the fact that $B_{i}$ are integers, we obtain

$$
\begin{aligned}
& \langle\tilde{\delta} \mid \tilde{\epsilon}\rangle\langle\tilde{\delta} \mid \tilde{\eta}\rangle\langle\tilde{\delta} \mid \tilde{\kappa}\rangle \epsilon^{4}\left(\epsilon, M_{B}\right) \epsilon^{4}\left(\eta, M_{B}\right) \epsilon^{4}\left(\kappa, M_{B}\right) \\
& =\epsilon^{4}\left(\delta, M_{B}\right)\langle\delta \mid \epsilon\rangle\langle\delta \mid \eta\rangle\langle\delta \mid \kappa\rangle \\
& \quad \times \exp \left\{-2 \pi i \sum_{i=1}^{h}\left(\delta_{i}^{\prime}\left(2 \delta_{i}^{\prime}+1\right)+\epsilon_{i}^{\prime}\left(2 \epsilon_{i}^{\prime}+1\right)+\eta_{i}^{\prime}\left(2 \eta_{i}^{\prime}+1\right)+\kappa_{i}^{\prime}\left(2 \kappa_{i}^{\prime}+1\right)\right) B_{i}\right\}
\end{aligned}
$$


Now, in the last exponential factor, the combinations $\delta_{i}^{\prime}\left(2 \delta_{i}^{\prime}+1\right)$ are integers whenever $\delta_{i}^{\prime}$ are half-integers, and thus the exponential factor is 1. Q.E.D.

It follows from Theorem 3 that

$$
\vartheta[\tilde{\delta}]^{4}(0, \tilde{\Omega}) \Xi_{6}^{\#}[\tilde{\delta}](\tilde{\Omega})=\operatorname{det}(C \Omega+D)^{8} \vartheta[\delta]^{4}(0, \Omega) \Xi_{6}^{\#}[\delta](\Omega)
$$

and hence the expression $\sum_{\delta} \Xi_{6}^{\#}[\delta] \vartheta[\delta]^{4}$ is a modular form of weight 8 . More precisely, define the modular form $\Psi_{4 k}(\Omega)$ by

$$
\Psi_{4 k}(\Omega)=\sum_{\delta} \vartheta[\delta](0, \Omega)^{8 k}
$$

For genus 2, where a complete classification of the polynomial ring of modular forms exists 6], these modular forms are subject to relations such as $4 \Psi_{8}=\Psi_{4}^{2}$. In higher genus, we have not found any analogous discussion in the literature. Remarkably, we have the following,

Theorem 4 The form $\Xi_{6}^{\#}[\delta](\Omega)$ satisfies the following identity in any genus $h \geq 2$

$$
\sum_{\delta} \Xi_{6}^{\#}[\delta] \vartheta[\delta]^{4}=-2^{2 h-5}\left(2^{h} \Psi_{8}-\Psi_{4}^{2}\right)
$$

Proof. In fact, we shall prove a more general result for the following more general quantity,

$$
\begin{aligned}
\Xi_{6}^{A}[\delta] \equiv-\frac{1}{2^{5} \cdot 3 !} \sum_{\delta_{1}, \delta_{2}, \delta_{3}} & \left\langle\delta \mid \delta_{1}\right\rangle\left\langle\delta \mid \delta_{2}\right\rangle\left\langle\delta \mid \delta_{3}\right\rangle \vartheta\left[\delta_{1}\right]^{4} \vartheta\left[\delta_{2}\right]^{4} \vartheta\left[\delta_{3}\right]^{4} \\
& \times\left(1+a_{0} e\left(\delta_{1}, \delta_{2}, \delta_{3}\right)\right)\left(1+a_{1} e\left(\delta, \delta_{2}, \delta_{3}\right)\right) \\
& \times\left(1+a_{2} e\left(\delta, \delta_{1}, \delta_{3}\right)\right)\left(1+a_{3} e\left(\delta, \delta_{1}, \delta_{2}\right)\right)
\end{aligned}
$$

Here, $A$ is an array of sign assignments $A=\left[a_{0} ; a_{1}, a_{2}, a_{3}\right]$, with $a_{0}, a_{1}, a_{2}, a_{3}= \pm 1$. Since $e\left(\delta_{1}, \delta_{2}, \delta_{3}\right) e\left(\delta, \delta_{1}, \delta_{2}\right) e\left(\delta, \delta_{1}, \delta_{3}\right) e\left(\delta, \delta_{2}, \delta_{3}\right)=+1$, it follows that $\Xi_{6}^{A}$ is non-vanishing only if $a_{0} a_{1} a_{2} a_{3}=+1$, which we henceforth assume. Two $A$ 's are equivalent if they differ by a permutation of their last 3 entries. The sign factor $\left\langle\delta \mid \delta_{1}\right\rangle\left\langle\delta \mid \delta_{2}\right\rangle\left\langle\delta \mid \delta_{3}\right\rangle$ would appear to involve a specific choice. Actually, all other choices are equivalent to it. For example, another modular covariant choice would be,

$$
\left\langle\delta \mid \delta_{1}\right\rangle\left\langle\delta_{2} \mid \delta_{3}\right\rangle=\left\langle\delta \mid \delta_{1}\right\rangle\left\langle\delta \mid \delta_{2}\right\rangle\left\langle\delta \mid \delta_{3}\right\rangle e\left(\delta, \delta_{2}, \delta_{3}\right)=a_{1}\left\langle\delta \mid \delta_{1}\right\rangle\left\langle\delta \mid \delta_{2}\right\rangle\left\langle\delta \mid \delta_{3}\right\rangle
$$

But, since $a_{1}$ is independent of $\delta_{i}$, this choice is equivalent to the one in (3.12). As a special case, we have $\Xi_{6}^{\#}[\delta]=\Xi_{6}^{A_{-}}[\delta]$ for $A_{-}=[-;-,-,-]$. 
Using the relation $a_{0} a_{1} a_{2} a_{3}=+1$, the form of $\Xi_{6}^{A}[\delta]$ may be simplified as follows,

$$
\begin{gathered}
\Xi_{6}^{A}[\delta]=-\frac{1}{96} \sum_{\delta_{1}, \delta_{2}, \delta_{3}}\left\langle\delta \mid \delta_{1}\right\rangle\left\langle\delta \mid \delta_{2}\right\rangle\left\langle\delta \mid \delta_{3}\right\rangle \vartheta\left[\delta_{1}\right]^{4} \vartheta\left[\delta_{2}\right]^{4} \vartheta\left[\delta_{3}\right]^{4}\left(1+a_{0} e\left(\delta_{1}, \delta_{2}, \delta_{3}\right)\right) \\
\times\left(1+a_{1} e\left(\delta, \delta_{2}, \delta_{3}\right)+a_{2} e\left(\delta, \delta_{1}, \delta_{3}\right)+a_{3} e\left(\delta, \delta_{1}, \delta_{2}\right)\right)
\end{gathered}
$$

We analyze this sum according to its $a_{i}$-dependence. The term independent of $a_{i}$ is proportional to $\vartheta[\delta]^{12}$ by the Riemann identities. In the term proportional to $a_{1}$, we use $\left\langle\delta \mid \delta_{1}\right\rangle\left\langle\delta \mid \delta_{2}\right\rangle\left\langle\delta \mid \delta_{3}\right\rangle e\left(\delta, \delta_{2}, \delta_{3}\right)=\left\langle\delta \mid \delta_{1}\right\rangle\left\langle\delta_{2} \mid \delta_{3}\right\rangle$. The sums over $\delta_{2}, \delta_{3}$ yield $\Psi_{4}$ by the Riemann identities, while the sum over $\delta_{1}$ yields $\vartheta[\delta]^{4}$. In the term proportional to $a_{0} a_{1}$, we use

$$
\left\langle\delta \mid \delta_{1}\right\rangle\left\langle\delta \mid \delta_{2}\right\rangle\left\langle\delta \mid \delta_{3}\right\rangle e\left(\delta_{1}, \delta_{2}, \delta_{3}\right) e\left(\delta, \delta_{2}, \delta_{3}\right)=\left\langle\delta_{1} \mid \delta\right\rangle\left\langle\delta_{1} \mid \delta_{2}\right\rangle\left\langle\delta_{1} \mid \delta_{3}\right\rangle
$$

The sums over $\delta_{2}$ and $\delta_{3}$ yield $\vartheta\left[\delta_{1}\right]^{8}$ and the remaining sum over $\delta_{1}$ is a sum over $\vartheta\left[\delta_{1}\right]^{12}$. In summary, we have the following simplified form, valid for general genus $h$,

$$
\begin{aligned}
\Xi_{6}^{A}[\delta]= & -\frac{1}{3} 2^{3 h-5}\left(1+a_{0}\right) \vartheta[\delta]^{12}-\frac{1}{3} 2^{2 h-5} a\left\{\vartheta[\delta]^{4} \Psi_{4}+a_{0} \sum_{\delta_{1}}\left\langle\delta \mid \delta_{1}\right\rangle \vartheta\left[\delta_{1}\right]^{12}\right\} \\
& -\frac{a_{0}}{48} \sum_{\delta_{1}, \delta_{2}, \delta_{3}}\left(1-e\left(\delta_{1}, \delta_{2}, \delta_{3}\right)\right)\left\langle\delta \mid \delta_{1}\right\rangle\left\langle\delta \mid \delta_{2}\right\rangle\left\langle\delta \mid \delta_{3}\right\rangle \vartheta\left[\delta_{1}\right]^{4} \vartheta\left[\delta_{2}\right]^{4} \vartheta\left[\delta_{3}\right]^{4}
\end{aligned}
$$

where we use the notation $a=a_{1}+a_{2}+a_{3}$.

The sum $\sum_{\delta} \Xi_{6}^{A}[\delta] \vartheta[\delta]^{4}$ may be calculated in terms of $\Psi_{4}$ and $\Psi_{8}$ using the Riemann relations, in addition to one further result, which involves the last term in (3.15). Concentrating on the summation over $\delta$, we have to carry out the following sum,

$$
\sum_{\delta}\left\langle\delta \mid \delta_{1}\right\rangle\left\langle\delta \mid \delta_{2}\right\rangle\left\langle\delta \mid \delta_{3}\right\rangle \vartheta[\delta](0)^{4}=\sum_{\delta}\left\langle\delta \mid \delta_{1}+\delta_{2}+\delta_{3}\right\rangle \vartheta[\delta](0)^{4}
$$

Using the fact that the triplet $\left(\delta_{1}, \delta_{2}, \delta_{3}\right)$ is restricted to be asyzygous by the projection prefactor in the last term of (3.15), the spin structure $\delta_{1}+\delta_{2}+\delta_{3}$ must be odd, and therefore, using the Riemann identities on the $\delta$-sum, (3.16) cancels for all $h \geq 2$. The final expression is then

$$
\sum_{\delta} \Xi_{6}^{A}[\delta] \vartheta[\delta]^{4}=-\frac{1}{3} 2^{3 h-5}\left(1+a_{0}\right)(1+a) \Psi_{8}-\frac{1}{3} 2^{2 h-5} a\left(\Psi_{4}^{2}-2^{h} \Psi_{8}\right)
$$

In the special case of $A=A_{-}$, we have $a_{0}=-1$ and $a=-3$, and we readily recover the statement of Theorem 3. Q.E.D.

Note that with the help of $\Xi_{6}^{\#}[\delta]$, the last sum in (3.15) may be eliminated, so that

$$
\begin{aligned}
\Xi_{6}^{A}[\delta]= & -a_{0} \Xi_{6}^{\#}[\delta]-\frac{1}{3} \cdot 2^{3 h-5}\left(1+a_{0}\right) \vartheta[\delta]^{12}+2^{2 h-5}\left(a_{0}-\frac{1}{3} a\right) \vartheta[\delta]^{4} \Psi_{4} \\
& -\frac{1}{3} \cdot 2^{2 h-5} a_{0}(a+3) \sum_{\delta_{1}}\left\langle\delta \mid \delta_{1}\right\rangle \vartheta\left[\delta_{1}\right]^{12} .
\end{aligned}
$$




\section{A conjectured identity between $\Psi_{8}(\Omega)$ and $\Psi_{4}(\Omega)$}

The identity in Theorem 3 suggests that the following relation between $\Psi_{8}(\Omega)$ and $\Psi_{4}(\Omega)$ should hold in any genus

$$
2^{h} \Psi_{8}(\Omega)-\Psi_{4}^{2}(\Omega)=0
$$

We note that $\Psi_{4 k}(\Omega) \rightarrow 2^{h}$ for all $k$ as $\operatorname{Im}\left(\Omega_{I I}\right) \rightarrow+i \infty$. This number simply corresponds to the $2^{h}$ spin structures for which $\delta_{I}^{\prime}=0$. This explains why the factor of $2^{h}$ in the relation between $\Psi_{4}$ and $\Psi_{8}$ is needed. In genus 1, the relation (4.1) corresponds to the well-known isospectrality of the root lattices of $E_{8} \times E_{8}$ and $S O(32)$, and follows immediately from the Jacobi identity $\vartheta_{3}^{4}=\vartheta_{2}^{4}+\vartheta_{4}^{4}$. In genus 2, it is a consequence of Igusa's classification of genus 2 modular forms. To the best of our knowledge, the relation has not been established for genus $h \geq 3$, although there is an extensive literature on related topics (see e.g. [12, and references therein). We present here some strong evidence for it in genus 3. For this, it is useful to define the following parameters for the period matrix $\Omega$

$$
\Omega=\left(\begin{array}{ccc}
\tau_{1} & \frac{1}{2} \sigma_{3} & \frac{1}{2} \sigma_{2} \\
\frac{1}{2} \sigma_{3} & \tau_{2} & \frac{1}{2} \sigma_{1} \\
\frac{1}{2} \sigma_{2} & \frac{1}{2} \sigma_{1} & \tau_{3}
\end{array}\right) \quad\left\{\begin{array} { l } 
{ x = e ^ { i \pi \tau _ { 1 } } } \\
{ y = e ^ { i \pi \tau _ { 2 } } } \\
{ z = e ^ { i \pi \tau _ { 3 } } }
\end{array} \quad \left\{\begin{array}{l}
u=e^{i \pi \sigma_{1}} \\
v=e^{i \pi \sigma_{2}} \\
w=e^{i \pi \sigma_{3}}
\end{array}\right.\right.
$$

\subsection{Asymptotic evidence}

The first type of evidence for the relation (4.1) is its vanishing asymptotics under completely separating degenerations. These correspond to $\sigma_{i} \rightarrow 0$. More precisely, we shall show that (4.1) holds for arbitrary $x, y, z$ up to and including the orders $\sigma_{1} \sigma_{2} \sigma_{3}$ and $\sigma_{1}^{2} \sigma_{2}^{2}, \sigma_{2}^{2} \sigma_{3}^{2}, \sigma_{1}^{2} \sigma_{3}^{2}$.

Now the $\vartheta$-function for the genus 3 period matrix can be written as

$$
\vartheta[\delta](z, \Omega)=\exp \left\{\frac{1}{4 \pi i}\left(\sigma_{3} \partial_{1} \partial_{2}+\sigma_{3} \partial_{1} \partial_{2}+\sigma_{3} \partial_{1} \partial_{2}\right)\right\} \prod_{i=1,2,3} \vartheta\left[\delta_{i}\right]\left(z_{i}, \tau_{i}\right)
$$

where $\delta^{t}=\left(\delta_{1}\left|\delta_{2}\right| \delta_{3}\right)^{t}, z=\left(z_{1}, z_{2}, z_{3}\right)$ and $\partial_{i}=\partial / \partial z_{i}$. Expanding the exponential into a series, we see that even and odd $\delta_{i}$ behave differently. We distinguish 2 types of even spin structures $\delta$ : those for which all genus 1 components carry even spin structures $\delta_{i}$, and those for which one component carries an even genus 1 spin structure but two components carry the odd spin structure. For the latter, $\vartheta[\delta]$ vanishes to order $\sigma_{i} \sigma_{j}$, which implies that the lowest order contribution to $\Psi_{4}$ and $\Psi_{8}$ that is generated by these terms is $\sigma_{i}^{8} \sigma_{j}^{8}$. As long as we work to orders less than 8 , it will thus be necessary to consider only spin structures $\delta$ for which all genus 1 components $\delta_{i}$ are even. 
Taking then all $\delta_{i}$ to be even and $z_{i}=0$, and using the heat equation, $\partial_{i}^{2} \vartheta\left[\delta_{i}\right]\left(z_{i}, \tau_{i}\right)=$ $4 \pi i \partial_{\tau_{i}} \vartheta\left[\delta_{i}\right]\left(z_{i}, \tau_{i}\right)$, we deduce the following relations,

$$
\begin{aligned}
\vartheta[\delta](0, \Omega)= & \sum_{k_{i}=0}^{\infty} \frac{\sigma_{1}^{2 k_{1}} \sigma_{2}^{2 k_{2}} \sigma_{3}^{2 k_{3}}}{\left(2 k_{1}\right) !\left(2 k_{2}\right) !\left(2 k_{3}\right) !} \partial_{\tau_{1}}^{k_{2}+k_{3}} \vartheta\left[\delta_{1}\right] \partial_{\tau_{2}}^{k_{1}+k_{3}} \vartheta\left[\delta_{2}\right] \partial_{\tau_{3}}^{k_{1}+k_{2}} \vartheta\left[\delta_{3}\right] \\
& +\sum_{k_{i}=0}^{\infty} \frac{\sigma_{1}^{2 k_{1}+1} \sigma_{2}^{2 k_{2}+1} \sigma_{3}^{2 k_{3}+1}}{\left(2 k_{1}+1\right) !\left(2 k_{2}+1\right) !\left(2 k_{3}+1\right) !} \partial_{\tau_{1}}^{k_{2}+k_{3}+1} \vartheta\left[\delta_{1}\right] \partial_{\tau_{2}}^{k_{1}+k_{3}+1} \vartheta\left[\delta_{2}\right] \partial_{\tau_{3}}^{k_{1}+k_{2}+1} \vartheta\left[\delta_{3}\right]
\end{aligned}
$$

where we have set $\partial_{\tau_{i}}^{k} \vartheta\left[\delta_{i}\right]=\partial_{\tau_{i}}^{k} \vartheta\left[\delta_{i}\right]\left(0, \tau_{i}\right)$. To linear order in $\sigma^{1}$, the first term occurring is obtained from the second term in the sum with $k_{1}=k_{2}=k_{3}=0$. Its contribution to $\Psi_{4}$ and $\Psi_{8}$ is respectively,

$$
\begin{aligned}
& \Psi_{4}=\sum_{i, j, k} \vartheta_{i}^{8}\left(\tau_{1}\right) \vartheta_{j}^{8}\left(\tau_{2}\right) \vartheta_{k}^{8}\left(\tau_{3}\right)\left\{1+8 \sigma_{1} \sigma_{2} \sigma_{3} \partial_{\tau_{1}} \ln \vartheta_{i} \partial_{\tau_{2}} \ln \vartheta_{j} \partial_{\tau_{3}} \ln \vartheta_{k}\right\} \\
& \Psi_{8}=\sum_{i, j, k} \vartheta_{i}^{16}\left(\tau_{1}\right) \vartheta_{j}^{16}\left(\tau_{2}\right) \vartheta_{k}^{16}\left(\tau_{3}\right)\left\{1+16 \sigma_{1} \sigma_{2} \sigma_{3} \partial_{\tau_{1}} \ln \vartheta_{i} \partial_{\tau_{2}} \ln \vartheta_{j} \partial_{\tau_{3}} \ln \vartheta_{k}\right\}
\end{aligned}
$$

Using now the genus 1 relation $\left(\sum_{i} \vartheta_{i}^{8}\right)^{2}=2 \sum_{i} \vartheta_{i}^{16}$, we readily see that the equation $\Psi_{4}^{2}=8 \Psi_{8}$ is obeyed at genus 3 to this order.

The next order is of order $\sigma_{1}^{2} \sigma_{2}^{2}$ (and by symmetry, the terms of order $\sigma_{1}^{2} \sigma_{3}^{2}$ and $\sigma_{2}^{2} \sigma_{3}^{2}$ are dealt with in the same way). The contributions to both modular forms are as follows,

$$
\begin{aligned}
& \Psi_{4}=\sum_{i, j, k} \vartheta_{i}^{8}\left(\tau_{1}\right) \vartheta_{j}^{8}\left(\tau_{2}\right) \vartheta_{k}^{8}\left(\tau_{3}\right)\left\{1+4 \sigma_{1}^{2} b_{j} c_{k}+4 \sigma_{2}^{2} a_{i} c_{k}+\sigma_{1}^{2} \sigma_{2}^{2} a_{i} b_{j}\left(2 c_{k}^{\prime}+14\left(c_{k}\right)^{2}\right\}\right. \\
& \Psi_{8}=\sum_{i, j, k} \vartheta_{i}^{16}\left(\tau_{1}\right) \vartheta_{j}^{16}\left(\tau_{2}\right) \vartheta_{k}^{16}\left(\tau_{3}\right)\left\{1+1+8 \sigma_{1}^{2} b_{j} c_{k}+8 \sigma_{2}^{2} a_{i} c_{k}+\sigma_{1}^{2} \sigma_{2}^{2} a_{i} b_{j}\left(4 c_{k}^{\prime}+60\left(c_{k}\right)^{2}\right\}\right.
\end{aligned}
$$

with the abbreviations, $a_{i}=\partial_{\tau_{1}} \vartheta_{i}\left(\tau_{1}\right) / \vartheta_{i}\left(\tau_{1}\right), b_{i}=\partial_{\tau_{2}} \vartheta_{i}\left(\tau_{2}\right) / \vartheta_{i}\left(\tau_{2}\right), c_{i}=\partial_{\tau_{3}} \vartheta_{i}\left(\tau_{3}\right) / \vartheta_{i}\left(\tau_{3}\right)$, $c_{i}^{\prime}=\partial_{\tau_{3}}^{2} \vartheta_{i}\left(\tau_{3}\right) / \vartheta_{i}\left(\tau_{3}\right)$. Next, we use the following formulas valid in genus 1 ,

$$
\sum_{i} \vartheta_{i}\left(\tau_{1}\right)^{8} a_{i}=\frac{1}{8} \partial_{\tau_{1}} \Psi_{4}\left(\tau_{1}\right) \quad \sum_{i} \vartheta_{i}\left(\tau_{1}\right)^{16} a_{i}=\frac{1}{16} \partial_{\tau_{1}} \Psi_{8}\left(\tau_{1}\right)
$$

and similar formulas for $b_{i}$ and $c_{i}$. Putting all together, we have

$$
\begin{aligned}
8 \Psi_{8}-\Psi_{4}^{2}= & \frac{\sigma_{1}^{2} \sigma_{2}^{2}}{256} \Psi_{4}\left(\tau_{1}\right)^{2} \Psi_{4}\left(\tau_{2}\right)^{2} \partial_{\tau_{1}} \Psi_{4}\left(\tau_{1}\right) \partial_{\tau_{2}} \Psi_{4}\left(\tau_{2}\right)\left\{-2 \Psi_{4}^{2}\left(\partial_{\tau_{3}} \Psi_{4}\right)^{2}\right. \\
& \left.+\sum_{k}\left[\vartheta_{k}\left(\tau_{3}\right)^{16}\left(32 c_{k}^{\prime}+480\left(c_{k}\right)^{2}\right)-\vartheta_{k}\left(\tau_{3}\right)^{4} \Psi_{4}\left(\tau_{3}\right)\left(16 c_{k}^{\prime}+112\left(c_{k}\right)^{2}\right)\right]\right\}
\end{aligned}
$$

Finally, using the relation

$$
\partial_{\tau_{3}}^{2}\left(2 \Psi_{8}\left(\tau_{3}\right)-\Psi_{4}\left(\tau_{3}\right)^{2}\right)=0
$$


the above contributions cancel, giving the asymptotics we claimed.

There is also evidence for the relation (4.1) in a different asymptotic region, namely in an expansion of $2^{h} \Psi_{8}(\Omega)-\Psi_{4}(\Omega)^{2}$ in powers of $x, y, z$, but exact in $u, v, w$. A $2 \mathrm{hrs}$ calculation using Maple 9 shows that the relation holds up to and including terms of order 4 in each variable, i.e. up to and including terms $x^{4}, y^{4}, z^{4}, x^{4} y^{4}, x^{4} z^{4}, y^{4} z^{4}$ and $x^{4} y^{4} z^{4}$.

\subsection{Evidence from special cases}

For general $x, y, z, w$ and $u=v=1$, (or $u=w=1$ or $v=w=1$ ) the relation follows from the corresponding properties at genus 2 , as well as from the relation $\Psi_{4}^{2}=2 \Psi_{8}$ at genus 1 .

\subsection{Numerical evidence}

The genus $3 \vartheta$-constants were computed numerically, up to and including 50 significant digits at random points in moduli space (this means picking points and verifying that $\operatorname{Im} \Omega>0$ ). The Riemann relations are then verified numerically and were found to agree to approximately 50 digits (recall that there are 36 terms, so a systematic error would cumulate in the sum and we should expect accuracy to 48 significant digits). Finally, the relations (4.1) was verified numerically. It was found to hold within about 48 digits. This has been carried out for seven points in moduli space; six with purely imaginary $\Omega$ and one with complex $\Omega$. The values of the points in moduli space were chosen so that $\operatorname{Im} \Omega>0$. Notice that real $x, y, z, u, v, w$ corresponds to purely imaginary $\Omega$. Numerically, this is the situation when the fewest cancellations occur and the highest precision can be expected.

\begin{tabular}{|c|c|c|c|c|c||c|c|}
\hline$x$ & $y$ & $z$ & $u$ & $v$ & $w$ & $\Psi_{4}$ & $\left|\Psi_{8}-\Psi_{4}^{2} / 8\right|$ \\
\hline \hline 0.3 & 0.4 & 0.5 & 1.1 & 1.2 & 1.4 & $5.75 \cdot 10^{6}$ & $<10^{-35}$ \\
\hline 0.3 & 0.4 & 0.5 & 1.1 & 1.2 & 1.3 & $4.68 \cdot 10^{6}$ & $<10^{-35}$ \\
\hline 0.3 & 0.4 & 0.5 & 1.1 & 1.2 & 1.2 & $3.98 \cdot 10^{6}$ & $<10^{-35}$ \\
\hline 0.3 & 0.4 & 0.5 & 1.1 & 1.2 & 1.1 & $3.56 \cdot 10^{6}$ & $<10^{-36}$ \\
\hline 0.02 & 0.04 & 0.05 & 0.11 & 0.12 & 0.14 & 72.1 & $<10^{-47}$ \\
\hline 0.0468 & 0.0666 & .0734 & .962 & 1.11 & 1.25 & 7.72 & $<10^{-46}$ \\
\hline $.0468+.1 i$ & $.0666-.2 i$ & .0734 & .962 & $1.11-.07 i$ & 1.25 & $27.6-23.6 i$ & $<10^{-45}$ \\
\hline
\end{tabular}

Table 2: Numerical evidence for $\Psi_{4}=8 \Psi_{8}$ in genus 3 


\section{$5 \quad$ Degeneration limits}

The behavior of a holomorphic modular covariant form near the divisor of Riemann surfaces with nodes is usually of considerable interest. For example, before the Gliozzi-Scherk-Olive projection, the superstring measure must exhibit a double pole, due to the presence of the tachyon (see e.g. 13] and [10] for a detailed discussion). In this section, we shall analyze the behavior of $\Xi_{6}^{\#}$ in the case of genus 3, when the underlying surface $\Sigma$ separates into a genus 1 surface $\Sigma^{(1)}$ and a genus 2 surface $\Sigma^{(2)}$.

Let $\left(A_{I}, B_{I}\right), 1 \leq I \leq 3$, be a canonical homology basis for the genus 3 surface $\Sigma$. Assume that the basis splits up in an obvious way, with $\left(A_{1}, A_{2} ; B_{1}, B_{2}\right)$ associated with the genus two surface while $\left(A_{3}, B_{3}\right)$ is associated with the genus 1 surface. The period matrix $\Omega_{I J}^{(3)}, I, J=1,2,3$ may be parametrized as follows,

$$
\Omega^{(3)}=\left(\begin{array}{ccc}
\Omega_{11} & \Omega_{12} & \tau_{1} \\
\Omega_{21} & \Omega_{22} & \tau_{2} \\
\tau_{1} & \tau_{2} & \Omega_{33}
\end{array}\right)
$$

and in the separating limit described above, we have $\tau_{1}, \tau_{2} \rightarrow 0$, while $\Omega_{33}$ and $\left\{\Omega_{I J}\right\}_{1 \leq I, J \leq 2}$ tend to the period matrices $\tau$ and $\Omega$ of $\Sigma^{(1)}$ and $\Sigma^{(2)}$.

It is natural to parametrize genus 3 spin structures $\Delta$ following the decomposition of the homology. Even spin structure fall into two classes $\left\{\Delta_{i a}\right\}$ and $\left\{\Delta_{m 0}\right\}$, according to whether the spin structures on the disconnected components is even/even or odd/odd,

$$
\Delta_{i a} \equiv\left(\begin{array}{c}
\delta_{i} \\
\mu_{a}
\end{array}\right) \quad \Delta_{m 0} \equiv\left(\begin{array}{c}
\nu_{m} \\
\mu_{0}
\end{array}\right)
$$

Here, $a=1,2,3$ runs over the even spin structures at genus $1, i=0,1, \cdots, 9$ runs over the even spin structures of genus 2 and $m=1, \cdots, 6$ runs over the odd spin structures of genus 2. To save notation, we denote the unique odd spin structure of genus 1 by $\mu_{0}$, instead of $\nu_{0}$ as previously. The genus $3 \vartheta$-constants (non-zero only for even spin structures) have a very simple behavior under the separating degeneration limit:

$$
\begin{aligned}
\vartheta\left[\Delta_{i a}\right](0, \Omega) & \rightarrow \vartheta_{2}\left[\delta_{i}\right]\left(0, \Omega_{2}\right) \cdot \vartheta_{1}\left[\mu_{a}\right]\left(0, \tau_{3}\right) \equiv \vartheta_{2}\left[\delta_{i}\right] \vartheta_{1}\left[\mu_{a}\right] \\
\vartheta\left[\Delta_{m 0}\right](0, \Omega) & \rightarrow 0 .
\end{aligned}
$$

Thus, to study the leading behavior of the $\vartheta$ constants at genus 3 , we shall be interested only in the spin structures $\Delta_{i a}$ and not $\Delta_{m 0}$. 
Theorem 5 Under a separating degeneration into a genus 2 and a genus 1 surface, the limit of the genus 3 form $\Xi_{6}^{\#}[\Delta]\left(\Omega^{(3)}\right)$ for an even spin structure $\Delta$ with even/even factorization on each disconnected component is given by

$$
\Xi_{6}^{\#}[\Delta]\left(\Omega^{(3)}\right)=F(\tau) \Xi_{6}[\delta](\Omega)+16 \dot{\eta}(\tau)^{12}\left\{2 \Lambda_{A}[\delta](\Omega)+\Lambda_{S}[\delta](\Omega)\right\} .
$$

Here $\delta$ is the spin structure component of $\Delta$ on the genus 2 surface, $F(\tau)$ is the genus 1 form defined by

$$
F(\tau)=\vartheta_{2}(\tau)^{12}+\vartheta_{3}(\tau)^{12}+\vartheta_{4}(\tau)^{12}+3 \vartheta_{4}(\tau)^{4} \vartheta_{3}(\tau)^{8}+3 \vartheta_{3}(\tau)^{4} \vartheta_{2}(\tau)^{8}+3 \vartheta_{3}(\tau)^{4} \vartheta_{4}(\tau)^{8}
$$

and $\Lambda_{A}[\delta](\Omega), \Lambda_{S}[\delta](\Omega)$ are the genus 2 forms defined both by sums of the form

$$
\sum\langle\delta \mid \epsilon\rangle\langle\delta \mid \eta\rangle\langle\delta \mid \kappa\rangle \vartheta[\epsilon]^{4}(0, \Omega) \vartheta[\eta]^{4}(0, \Omega) \vartheta[\kappa]^{4}(0, \Omega),
$$

but over different sets of triplets $\{\epsilon, \eta, \kappa\}$ of even spin structures. For $\Lambda_{A}[\delta](\Omega)$, the triplet $\{\epsilon, \eta, \kappa\}$ is required to be asyzygous, while for $\Lambda_{S}[\delta](\Omega)$, it is required to be syzygous. For both, $\{\epsilon, \eta, \kappa\}$ is also required to combine with $\delta$ into a quartet which contains exactly 2 asyzygous triplets.

Proof. Under $S p(6, \mathbf{Z})$ modular transformations, we can map any even spin structure into $\Delta=(0 \mid 0)$, in which case $\Xi_{6}[\Delta]\left(\Omega^{(3)}\right)$ becomes

$$
\Xi_{6}^{\#}[\Delta]\left(\Omega^{(3)}\right)=-\frac{1}{2} \sum_{[\Delta, \epsilon, \eta, \kappa]} \vartheta[\epsilon]^{4}\left(0, \Omega^{(3)}\right) \vartheta[\eta]^{4}\left(0, \Omega^{(3)}\right) \vartheta[\kappa]^{4}\left(0, \Omega^{(3)}\right)
$$

In the degenerating limit, only spin structures of the form $\Delta_{i a}$ will survive, so we may immediately restrict $\epsilon, \eta, \kappa$ to be of that form,

$$
\epsilon_{i a} \equiv\left(\begin{array}{c}
\delta_{i} \\
\mu_{a}
\end{array}\right) \quad \eta_{j b} \equiv\left(\begin{array}{c}
\delta_{j} \\
\mu_{b}
\end{array}\right) \quad \kappa_{k c} \equiv\left(\begin{array}{c}
\delta_{k} \\
\mu_{c}
\end{array}\right)
$$

The asymptotics is now manifest,

$$
-2 \Xi_{6}^{\#}[\Delta]\left(\Omega^{(3)}\right) \rightarrow \sum_{\left[\delta_{11}, \epsilon_{i a}, \eta_{j b}, \kappa_{k c}\right]} \vartheta_{2}\left[\delta_{i}\right]^{4} \vartheta_{2}\left[\delta_{j}\right]^{4} \vartheta_{2}\left[\delta_{k}\right]^{4} \cdot \vartheta_{1}\left[\mu_{a}\right]^{4} \vartheta_{1}\left[\mu_{b}\right]^{4} \vartheta_{1}\left[\mu_{c}\right]^{4}
$$

It remains to identify the totally asyzygous quartets that contribute and to parametrize them in a convenient way. The conditions are

$$
\begin{aligned}
& e\left(\delta_{1}, \delta_{i}, \delta_{j}\right) \cdot e\left(\mu_{1}, \mu_{a}, \mu_{b}\right)=-1 \\
& e\left(\delta_{1}, \delta_{j}, \delta_{k}\right) \cdot e\left(\mu_{1}, \mu_{b}, \mu_{c}\right)=-1 \\
& e\left(\delta_{1}, \delta_{k}, \delta_{i}\right) \cdot e\left(\mu_{1}, \mu_{c}, \mu_{a}\right)=-1 \\
& e\left(\delta_{i}, \delta_{j}, \delta_{k}\right) \cdot e\left(\mu_{a}, \mu_{b}, \mu_{c}\right)=-1
\end{aligned}
$$


It is not hard to analyze the equation on the genus 1 spin structures. Denoting the choice of the 3 genus 1 spin structures simply by their numbers $a b c$, and listing the 4 values of $e$ above in a row vector, we have

$$
\begin{array}{llll}
111,222,333 & ++++ & 123,132 & +-+- \\
112,121,211 & ++++ & 231,321 & -++- \\
113,131,311 & ++++ & 312,213 & ++-- \\
122,212,221 & ++++ & 223,332 & +--+ \\
133,313,331 & ++++ & 232,323 & --++ \\
& & 322,233 & -+-+
\end{array}
$$

Thus, up to permutations, there are only two cases. The case where all signs are + produces genus 2 totally asyzygous quartets of the form $\left[\delta_{1}, \delta_{i}, \delta_{j}, \delta_{k}\right]$, since we have

$$
\begin{array}{ll}
e\left(\delta_{1}, \delta_{i}, \delta_{j}\right)=-1 & e\left(\mu_{1}, \mu_{a}, \mu_{b}\right)=+1 \\
e\left(\delta_{1}, \delta_{j}, \delta_{k}\right)=-1 & e\left(\mu_{1}, \mu_{b}, \mu_{c}\right)=+1 \\
e\left(\delta_{1}, \delta_{k}, \delta_{i}\right)=-1 & e\left(\mu_{1}, \mu_{c}, \mu_{a}\right)=+1 \\
e\left(\delta_{i}, \delta_{j}, \delta_{k}\right)=-1 & e\left(\mu_{a}, \mu_{b}, \mu_{c}\right)=+1
\end{array}
$$

Thus $\left\{\delta_{1}, \delta_{i}, \delta_{j}, \delta_{k}\right\}$ form a totally asyzygous quartet, and we recognize the structure of $\Xi_{6}\left[\delta_{1}\right]\left(\Omega^{(2)}\right)$. Furthermore, the fact that they are totally asyzygous implies that these spin structures must all be distinct. The corresponding contributions tend towards the first term on the right hand side of (5.4).

In the remaining contributions, with two + and two - signs in (5.11), the genus 1 part is actually always proportional to $\eta^{12}$. This is clear in the first three lines, where the product $\vartheta_{2}^{4} \vartheta_{3}^{4} \vartheta_{4}^{4}=16 \eta^{12}$ manifestly factors out. But it is also true in the last three lines, upon adding up the contributions from the two cases listed. For example,

$$
223 \rightarrow \vartheta_{2}^{8} \vartheta_{4}^{4} \quad 332 \rightarrow \vartheta_{2}^{4} \vartheta_{4}^{8}
$$

Their sum is $\vartheta_{2}^{8} \vartheta_{4}^{4}+\vartheta_{2}^{4} \vartheta_{4}^{8}=\vartheta_{2}^{4} \vartheta_{3}^{4} \vartheta_{4}^{4}=16 \eta^{12}$. It remains only to identify the genus 2 contribution. In the present case, the values $e\left(\delta_{1}, \delta_{i}, \delta_{j}\right), e\left(\delta_{1}, \delta_{j}, \delta_{k}\right), e\left(\delta_{1}, \delta_{i}, \delta_{k}\right), e\left(\delta_{i}, \delta_{j}, \delta_{k}\right)$ must contain exactly two signs + and two signs - i.e., the quartet $\left\{\delta_{1}, \delta_{i}, \delta_{j}, \delta_{k}\right\}$ contains exactly two syzygous triplets and two asyzygous triplets. These quartets come in two groups, one group characterized by the fact that $\left\{\delta_{i}, \delta_{j}, \delta_{k}\right\}$ is an asyzygous triplet, and the other characterized by the fact that $\left\{\delta_{i}, \delta_{j}, \delta_{k}\right\}$ is syzygous. Since the first three lines of (5.11) correspond to $\left\{\delta_{i}, \delta_{j}, \delta_{k}\right\}$ asyzygous and the next three to $\left\{\delta_{i}, \delta_{j}, \delta_{k}\right\}$ syzygous, Theorem 5 follows. Q.E.D. 


\section{A Modular transformations for any genus}

Modular transformations in genus $h$ form the infinite discrete group $S p(2 h, \mathbf{Z})$, defined by

$$
M=\left(\begin{array}{cc}
A & B \\
C & D
\end{array}\right) \quad\left(\begin{array}{cc}
A & B \\
C & D
\end{array}\right)\left(\begin{array}{cc}
0 & I \\
-I & 0
\end{array}\right)\left(\begin{array}{cc}
A & B \\
C & D
\end{array}\right)^{t}=\left(\begin{array}{cc}
0 & I \\
-I & 0
\end{array}\right)
$$

where $A, B, C, D$ are integer valued $h \times h$ matrices and the superscript ${ }^{t}$ denotes transposition. To exhibit the action of the modular group on $1 / 2$ characteristics $\kappa$ (even or odd), it is convenient to assemble the $1 / 2$ characteristics into a single column of $2 h$ entries and the action of the modular group is then given by [14]

$$
\left(\begin{array}{c}
\tilde{\kappa}^{\prime} \\
\tilde{\kappa}^{\prime \prime}
\end{array}\right)=\left(\begin{array}{cc}
D & -C \\
-B & A
\end{array}\right)\left(\begin{array}{c}
\kappa^{\prime} \\
\kappa^{\prime \prime}
\end{array}\right)+\frac{1}{2} \operatorname{diag}\left(\begin{array}{c}
C D^{T} \\
A B^{T}
\end{array}\right)
$$

Here and below, $\operatorname{diag}(M)$ of a $h \times h$ matrix $M$ is an $1 \times h$ column vector whose entries are the diagonal entries on $M$. On the period matrix, the transformation acts by

$$
\tilde{\Omega}=(A \Omega+B)(C \Omega+D)^{-1}
$$

while on the Jacobi $\vartheta$-functions, the action is given by

$$
\vartheta[\tilde{\kappa}]\left(\left\{(C \Omega+D)^{-1}\right\}^{t} \zeta, \tilde{\Omega}\right)=\epsilon(\kappa, M) \operatorname{det}(C \Omega+D)^{\frac{1}{2}} e^{i \pi \zeta^{t}(C \Omega+D)^{-1} C \zeta} \vartheta[\kappa](\zeta, \Omega)
$$

where $\kappa=\left(\kappa^{\prime} \mid \kappa^{\prime \prime}\right)$ and $\tilde{\kappa}=\left(\tilde{\kappa}^{\prime} \mid \tilde{\kappa}^{\prime \prime}\right)$. The phase factor $\epsilon(\kappa, M)$ depends upon both $\kappa$ and the modular transformation $M$ and obeys $\epsilon(\kappa, M)^{8}=1$.

\section{A.1 Convenient generators of the modular group}

Using a Lemma (15) of [7], the group is generated by

$$
\left(\begin{array}{cc}
A & B \\
0 & D
\end{array}\right) \quad \text { and } \quad\left(\begin{array}{cc}
0 & I \\
-I & 0
\end{array}\right)
$$

where $I$ is the $h \times h$ identity matrix. The first set is easily seen to be generated in turn by the subgroups generated by matrices of the form $M_{A}$ and $M_{B}$, so that the full $S p(2 h, \mathbf{Z})$ is generated by three subgroups,

$$
M_{A}=\left(\begin{array}{cc}
A & 0 \\
0 & \left(A^{t}\right)^{-1}
\end{array}\right) \quad M_{B}=\left(\begin{array}{cc}
I & B \\
0 & I
\end{array}\right) \quad S=\left(\begin{array}{cc}
0 & I \\
-I & 0
\end{array}\right)
$$

where $A \in S L(h, \mathbf{Z})$ and $B^{t}=B$, the latter forming the translation group $\mathbf{Z}^{\frac{1}{2} h(h+1)}$. The action on spin structures for each of the 3 subgroups is given by

$$
\begin{aligned}
M_{A}\left(\kappa^{\prime} \mid \kappa^{\prime \prime}\right) & =\left(\left(A^{t}\right)^{-1} \kappa^{\prime} \mid A \kappa^{\prime \prime}\right) \\
M_{B}\left(\kappa^{\prime} \mid \kappa^{\prime \prime}\right) & =\left(\kappa^{\prime} \mid A \kappa^{\prime \prime}-B \kappa^{\prime}+\frac{1}{2} \operatorname{diag} B\right) \\
S\left(\kappa^{\prime} \mid \kappa^{\prime \prime}\right) & =\left(\kappa^{\prime \prime} \mid \kappa^{\prime}\right)
\end{aligned}
$$




\section{A.2 Modular transformations of $\vartheta$-constants}

We shall be most interested in the modular transformations of $\vartheta$-constants of even spin structures $\delta$, which are given by

$$
\vartheta[\tilde{\delta}](0, \tilde{\Omega})=\epsilon(\delta, M) \operatorname{det}(C \Omega+D)^{\frac{1}{2}} \vartheta[\delta](0, \Omega)
$$

and we have

$$
\begin{aligned}
\epsilon\left(\delta, M_{A}\right) & =1 \\
\epsilon\left(\delta, M_{B}\right) & =\exp \left\{-i \pi \delta^{\prime t} B \delta^{\prime}+i \pi \delta^{\prime t} \operatorname{diag} B\right\} \\
\epsilon(\delta, S) & =\exp \{i \pi h / 4\}
\end{aligned}
$$

\section{A.3 Modular transformations of signatures}

The signature on pairs is not invariant under all modular transformations; instead,

$$
\begin{aligned}
\left\langle M_{A} \delta \mid M_{A} \epsilon\right\rangle & =\langle\delta \mid \epsilon\rangle \\
\left\langle M_{B} \delta \mid M_{B} \epsilon\right\rangle & =\langle\delta \mid \epsilon\rangle \cdot \exp \left\{2 \pi i\left(\delta^{\prime}-\epsilon^{\prime}\right)^{t} \operatorname{diag} B\right\} \\
\langle S \delta \mid S \epsilon\rangle & =\langle\delta \mid \epsilon\rangle
\end{aligned}
$$

This transformation law means, however, that the signature for triplets is invariant under all modular transformations. It suffices to check this for transformations $M_{B}$,

$$
\begin{aligned}
e\left(M_{B} \delta, M_{B} \epsilon, M_{B} \eta\right) & =\left\langle M_{B} \delta \mid M_{B} \epsilon\right\rangle\left\langle M_{B} \epsilon \mid M_{B} \eta\right\rangle\left\langle M_{B} \eta \mid M_{B} \delta\right\rangle \\
& =e(\delta, \epsilon, \eta)
\end{aligned}
$$

where the exponential factors arising from the transformation law of the three pairs cancel. In particular, totally asyzygous $N$-tuples are mapped into totally asyzygous $N$-tuples under modular transformations.

\section{B Spin structures and asyzygies in genus 2}

The spin structures for genus 2 Riemann surfaces are described in detail in [4, $§ 2.1-\S 2.3$. For the readers' convenience, we reproduce here the notation, since it is used extensively below. The odd spin structures may be labeled by

$$
\begin{aligned}
2 \nu_{1}=\left(\begin{array}{l|l}
0 & 0 \\
1 & 1
\end{array}\right) & 2 \nu_{3}=\left(\begin{array}{l|l}
0 & 1 \\
1 & 1
\end{array}\right) & 2 \nu_{5}=\left(\begin{array}{l|l}
1 & 0 \\
1 & 1
\end{array}\right) \\
2 \nu_{2}=\left(\begin{array}{l|l}
1 & 1 \\
0 & 0
\end{array}\right) & 2 \nu_{4}=\left(\begin{array}{l|l}
1 & 1 \\
0 & 1
\end{array}\right) & 2 \nu_{6}=\left(\begin{array}{l|l}
1 & 1 \\
1 & 0
\end{array}\right)
\end{aligned}
$$


and the even spin structures by

$$
\begin{array}{rlrl}
2 \delta_{1}=\left(\begin{array}{l|l}
0 & 0 \\
0 & 0
\end{array}\right) & 2 \delta_{2}=\left(\begin{array}{l|l}
0 & 0 \\
0 & 1
\end{array}\right) & 2 \delta_{3}=\left(\begin{array}{l|l}
0 & 1 \\
0 & 0
\end{array}\right) & 2 \delta_{4}=\left(\begin{array}{l|l}
0 & 1 \\
0 & 1
\end{array}\right) \\
2 \delta_{5}=\left(\begin{array}{l|l}
0 & 0 \\
1 & 0
\end{array}\right) & 2 \delta_{6}=\left(\begin{array}{l|l}
0 & 1 \\
1 & 0
\end{array}\right) & 2 \delta_{7}=\left(\begin{array}{l|l}
1 & 0 \\
0 & 0
\end{array}\right) & 2 \delta_{8}=\left(\begin{array}{l|l}
1 & 0 \\
0 & 1
\end{array}\right) \\
& 2 \delta_{9}=\left(\begin{array}{l|l}
1 & 0 \\
1 & 0
\end{array}\right) & 2 \delta_{0}=\left(\begin{array}{l|l}
1 & 1 \\
1 & 1
\end{array}\right)
\end{array}
$$

Generators of genus 2 modular transformations $M_{1}, M_{2}, M_{3}, \Sigma, T, S$ are chosen as they were defined in [4].

\section{B.1 Tables of asyzygies in genus 2}

The properties of asyzygies often require a case by case treatment. For genus 2, it is convenient to simply make full tables of asyzygies, and check the desired properties by inspection of these tables. In this Appendix, we provide the tables which have been used in this paper.

There are 120 distinct triples of which 60 are syzygous, $e=+1$,

$\begin{array}{llllllllll}(123) & (124) & (127) & (128) & (134) & (135) & (136) & (149) & (140) & (156) \\ (157) & (159) & (168) & (160) & (178) & (179) & (180) & (190) & (234) & (239) \\ (230) & (245) & (246) & (256) & (258) & (259) & (267) & (260) & (278) & (270) \\ (289) & (290) & (347) & (348) & (356) & (358) & (350) & (367) & (369) & (378) \\ (379) & (380) & (390) & (456) & (457) & (450) & (468) & (469) & (478) & (470) \\ (489) & (490) & (579) & (570) & (589) & (580) & (679) & (670) & (689) & (680)\end{array}$

and 60 are asyzygous, $e=-1$,

$\begin{array}{llllllllll}(125) & (126) & (129) & (120) & (137) & (138) & (139) & (130) & (145) & (146) \\ (147) & (148) & (158) & (150) & (167) & (169) & (170) & (189) & (235) & (236) \\ (237) & (238) & (247) & (248) & (249) & (240) & (257) & (250) & (268) & (269) \\ (279) & (280) & (345) & (346) & (349) & (340) & (357) & (359) & (368) & (360) \\ (370) & (389) & (458) & (459) & (467) & (460) & (479) & (480) & (567) & (568) \\ (569) & (560) & (578) & (590) & (678) & (690) & (789) & (780) & (790) & (890)\end{array}$




\section{B.2 Asyzygous quartets in genus 2}

The list of asyzygous quartets can be easily read off the table of asyzygous triplets given above. It turns out that there are 15 asyzygous quartets in all. They are given in the table below, together with their transformations under modular transformations.

\begin{tabular}{|c||c|c|c|c|c|c|}
\hline TAQ & $M_{1}$ & $M_{2}$ & $M_{3}$ & $S$ & $\Sigma$ & $T$ \\
\hline \hline 1250 & 3460 & 2150 & 1269 & 1520 & 1370 & 1458 \\
\hline 1269 & 3459 & 1269 & 1250 & 1584 & 1389 & 1467 \\
\hline 1370 & 1370 & 2480 & 1389 & 1730 & 1250 & 1398 \\
\hline 1389 & 1389 & 2479 & 1370 & 1467 & 1269 & 1370 \\
\hline 1458 & 2368 & 2357 & 1467 & 1269 & 1467 & 1250 \\
\hline 1467 & 2357 & 2368 & 1458 & 1389 & 1458 & 1269 \\
\hline 2357 & 1467 & 1458 & 2368 & 2357 & 2357 & 3459 \\
\hline 2368 & 1458 & 1467 & 2357 & 5678 & 2368 & 3460 \\
\hline 2479 & 2479 & 1389 & 2480 & 3459 & 3459 & 2497 \\
\hline 2480 & 2480 & 1370 & 2479 & 5690 & 3460 & 2480 \\
\hline 3459 & 1269 & 3459 & 3460 & 2479 & 2479 & 2357 \\
\hline 3460 & 1250 & 3460 & 3459 & 7890 & 2480 & 2368 \\
\hline 5678 & 5678 & 5678 & 5678 & 2368 & 5678 & 5690 \\
\hline 5690 & 5690 & 5690 & 5690 & 2480 & 7890 & 5678 \\
\hline 7890 & 7890 & 7890 & 7890 & 3460 & 5690 & 7890 \\
\hline
\end{tabular}

Table 3: Modular transformations of totally asyzygous quartets (TAQ)

\section{B.3 Admissible sextets in genus 2}

We consider sextets of even spin structures in genus 2 satisfying the property listed in Theorem 2: they can be written as the union of three pairs, the union of any two pairs forming a totally asyzygous quartet. For convenience, we sometimes refer to sextets with this propery as admissible sextets. In the table below, we have listed all the 15 sextets in genus 2 satisfying this property. The three totally asyzygous quartets that each sextet contains have been denoted by $A, B$, and $C$. The three pairs can then be recaptured by taking $A \cap B, B \cap C$, and $A \cap C$.

Given a fixed spin structure $\delta$, the admissible sextets split into two groups $s^{c}[\delta]$ and $s[\delta]$, characterized respectively as the sextets which include and do not include $\delta$. For each fixed $\delta, s[\delta]$ always consists of 6 sextets, and $s^{c}[\delta]$ of 9 sextets. In the table below, the first 6 sextets are the sextets in $s\left[\delta_{7}\right]$, and the remaining ones are the sextets in $s^{c}\left[\delta_{7}\right]$. 


\begin{tabular}{|c||c|c|c|c|c|c|c|}
\hline sextet & $M_{3} \epsilon^{2}$ & $S$ & $T$ & $\Sigma$ & $A \cap B$ & $B \cap C$ & $C \cap A$ \\
\hline \hline 123570 & $123689-$ & 123570 & 134589 & 123570 & 10 & 37 & 25 \\
\hline 123689 & $123570-$ & 145678 & 134670 & 123689 & 19 & 38 & 26 \\
\hline 124580 & $124679-$ & 125690 & 124580 & 134670 & 48 & 20 & 15 \\
\hline 124679 & $124580-$ & 134589 & 124679 & 134589 & 16 & 47 & 29 \\
\hline 125690 & $125690+$ & 124580 & 145678 & 137890 & 12 & 69 & 50 \\
\hline 134589 & $134670-$ & 124679 & 123570 & 124679 & 18 & 45 & 39 \\
\hline 134670 & $134589-$ & 137890 & 123689 & 124580 & 17 & 46 & 30 \\
\hline 137890 & $137890+$ & 134670 & 137890 & 125690 & 13 & 89 & 70 \\
\hline 145678 & $145678+$ & 123689 & 125690 & 145678 & 14 & 67 & 58 \\
\hline 234579 & $234680-$ & 234579 & 234579 & 234579 & 27 & 49 & 35 \\
\hline 234680 & $234579-$ & 567890 & 234680 & 234680 & 28 & 40 & 36 \\
\hline 235678 & $235678+$ & 235678 & 345690 & 235678 & 23 & 68 & 57 \\
\hline 247890 & $247890+$ & 345690 & 247890 & 345690 & 24 & 80 & 79 \\
\hline 345690 & $345690+$ & 247890 & 235678 & 247890 & 34 & 60 & 59 \\
\hline 567890 & $567890+$ & 234680 & 567890 & 567890 & 56 & 90 & 78 \\
\hline
\end{tabular}

Table 4: Modular transformations and TAQ structure of admissible sextets

\section{Genus 3 spin structures \& modular transf}

Here, we provide a summary of the computer results obtained on genus 3 spin structures, as well as multiplets of spin structures. The genus 3 spin structures are labeled as follows,

$$
\begin{aligned}
& \Delta_{1}=[0,0,0,0,0,0] \quad \Delta_{13}=[0,1,0,0,1,0] \quad \Delta_{25}=[1,0,0,1,0,1] \\
& \Delta_{2}=[0,0,0,0,0,1] \quad \Delta_{14}=[0,1,0,1,0,0] \quad \Delta_{26}=[1,0,0,1,1,0] \\
& \Delta_{3}=[0,0,0,0,1,0] \quad \Delta_{15}=[0,1,0,1,0,1] \quad \Delta_{27}=[1,0,1,0,0,0] \\
& \Delta_{4}=[0,0,0,1,0,0] \quad \Delta_{16}=[0,1,0,1,1,0] \quad \Delta_{28}=[1,0,1,0,0,1] \\
& \Delta_{5}=[0,0,0,1,0,1] \quad \Delta_{17}=[0,1,1,0,0,0] \quad \Delta_{29}=[1,0,1,0,1,0] \\
& \Delta_{6}=[0,0,0,1,1,0] \quad \Delta_{18}=[0,1,1,0,0,1] \quad \Delta_{30}=[1,0,1,1,1,1] \\
& \Delta_{7}=[0,0,1,0,0,0] \quad \Delta_{19}=[0,1,1,0,1,0] \quad \Delta_{31}=[1,1,0,0,1,1] \\
& \Delta_{8}=[0,0,1,0,0,1] \quad \Delta_{20}=[0,1,1,1,1,1] \quad \Delta_{32}=[1,1,0,1,1,1] \\
& \Delta_{9}=[0,0,1,0,1,0] \quad \Delta_{21}=[1,0,0,0,0,0] \quad \Delta_{33}=[1,1,1,0,1,1] \\
& \Delta_{10}=[0,0,1,1,1,1] \quad \Delta_{22}=[1,0,0,0,0,1] \quad \Delta_{34}=[1,1,1,1,0,0] \\
& \Delta_{11}=[0,1,0,0,0,0] \quad \Delta_{23}=[1,0,0,0,1,0] \quad \Delta_{35}=[1,1,1,1,0,1] \\
& \Delta_{12}=[0,1,0,0,0,1] \quad \Delta_{24}=[1,0,0,1,0,0] \quad \Delta_{36}=[1,1,1,1,1,0]
\end{aligned}
$$


and we take the following basis of genus 3 modular generators,

$$
B_{i}=\left(\begin{array}{cc}
I & b_{i} \\
0 & I
\end{array}\right) \quad A_{i}=\left(\begin{array}{cc}
a_{i} & 0 \\
0 & \left(a_{i}^{t}\right)^{-1}
\end{array}\right) \quad S=\left(\begin{array}{cc}
0 & I \\
-I & 0
\end{array}\right)
$$

where

$$
\begin{aligned}
& b_{1}=\left(\begin{array}{ccc}
1 & 0 & 0 \\
0 & 0 & 0 \\
0 & 0 & 0
\end{array}\right) \quad b_{2}=\left(\begin{array}{ccc}
0 & 0 & 0 \\
0 & 1 & 0 \\
0 & 0 & 0
\end{array}\right) \quad b_{3}=\left(\begin{array}{lll}
0 & 0 & 0 \\
0 & 0 & 0 \\
0 & 0 & 1
\end{array}\right) \\
& b_{4}=\left(\begin{array}{lll}
0 & 1 & 0 \\
1 & 0 & 0 \\
0 & 0 & 0
\end{array}\right) \quad b_{5}=\left(\begin{array}{ccc}
0 & 0 & 1 \\
1 & 0 & 0 \\
0 & 0 & 0
\end{array}\right) \quad b_{6}=\left(\begin{array}{lll}
0 & 0 & 0 \\
0 & 0 & 1 \\
0 & 1 & 0
\end{array}\right) \\
& a_{1}=\left(\begin{array}{ccc}
0 & 1 & 0 \\
-1 & 0 & 0 \\
0 & 0 & 1
\end{array}\right) \quad a_{2}=\left(\begin{array}{ccc}
1 & 0 & 0 \\
0 & 0 & 1 \\
0 & -1 & 0
\end{array}\right) \quad a_{3}=\left(\begin{array}{ccc}
0 & 0 & 1 \\
0 & 1 & 0 \\
-1 & 0 & 0
\end{array}\right) \\
& a_{4}=\left(\begin{array}{ccc}
1 & 1 & 0 \\
0 & 1 & 0 \\
0 & 0 & 1
\end{array}\right) \quad a_{5}=\left(\begin{array}{ccc}
1 & 0 & 1 \\
0 & 1 & 0 \\
0 & 0 & 1
\end{array}\right) \quad a_{6}=\left(\begin{array}{ccc}
1 & 0 & 0 \\
0 & 1 & 1 \\
0 & 0 & 1
\end{array}\right)
\end{aligned}
$$

The action of all elementary modular generators $\left(A_{i}, B_{i}, i=1, \cdots, 6\right.$ and $\left.S\right)$ are given in Table 5 below. The action of the additional generator $\Lambda \equiv B_{1} S B_{1} S B_{1}$ is also given. The generators $B_{4}, B_{5}, B_{6}, A_{1}, A_{2}, A_{3}, A_{4}, A_{5}, A_{6}, S$, and $\Lambda$ form a subgroup leaving $\Delta_{1}$ invariant. It is conjectured that these generators span the maximal subgroup of $S p\left(6, \mathbf{Z}_{2}\right)$ which leaves $\Delta_{1}$ invariant.

\section{C.1 Multiplets of spin structures}

Asyzygous combinations play an important role. An asyzygous triplet $\left(\lambda_{1}, \lambda_{2}, \lambda_{3}\right)$ is defined so that $e\left(\lambda_{1}, \lambda_{2}, \lambda_{3}\right)=-1$. An asyzygous $N$-plet (with $N>3$ ) is a set of spin structures such that each triplet of distinct spin structures is asyzygous. The count is as follows,

$\begin{aligned} \text { asyzygous quartets } & 5040 \\ \text { asyzygous quintets } & 2016 \\ \text { asyzygous sextets } & 336 \\ \text { asyzygous septets } & 0\end{aligned}$




\begin{tabular}{|c|c|c|c|c|c|c|c|c|c|c|c|c|c|c|}
\hline$\delta$ & $B_{1}$ & $B_{2}$ & $B_{3}$ & $B_{4}$ & $B_{5}$ & $B_{6}$ & $A_{1}$ & $A_{2}$ & $A_{3}$ & $A_{4}$ & $A_{5}$ & $A_{6}$ & $S$ & $\Lambda$ \\
\hline$\overline{c 1}$ & 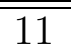 & $\overline{\overline{4}}$ & $\overline{\overline{2}}$ & $\overline{1}$ & $\overline{1}$ & 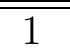 & $\overline{11}$ & $\overline{1}$ & $\overline{1}$ & $\overline{1}$ & 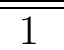 & $\bar{~} 1$ & $\overline{\overline{1}}$ & $\overline{11}$ \\
\hline 2 & 12 & 5 & 1 & 2 & 2 & 2 & 2 & 4 & 11 & 2 & 12 & 2 & 3 & 2 \\
\hline 3 & 13 & 6 & 3 & 3 & 13 & 6 & 3 & 7 & 21 & 3 & 3 & 9 & 2 & 3 \\
\hline 4 & 14 & 1 & 5 & 4 & 4 & 4 & 11 & 2 & 4 & 14 & 4 & 5 & 7 & 4 \\
\hline 5 & 15 & 2 & 4 & 5 & 5 & 5 & 12 & 5 & 14 & 15 & 15 & 4 & 9 & 5 \\
\hline 6 & 16 & 3 & 6 & 6 & 16 & 3 & 13 & 8 & 24 & 16 & 6 & 10 & 8 & 6 \\
\hline 7 & 17 & 7 & 8 & 17 & 7 & 8 & 21 & 3 & 7 & 7 & 7 & 7 & 4 & 7 \\
\hline 8 & 18 & 8 & 7 & 18 & 8 & 7 & 22 & 6 & 17 & 8 & 18 & 8 & 6 & 8 \\
\hline 9 & 19 & 9 & 9 & 19 & 19 & 10 & 23 & 9 & 27 & 9 & 9 & 3 & 5 & 9 \\
\hline 10 & 20 & 10 & 10 & 20 & 20 & 9 & 31 & 10 & 34 & 20 & 20 & 6 & 10 & 10 \\
\hline 11 & 1 & 14 & 12 & 11 & 11 & 11 & 4 & 11 & 2 & 11 & 11 & 11 & 21 & 21 \\
\hline 12 & 2 & 15 & 11 & 12 & 12 & 12 & 5 & 14 & 12 & 12 & 2 & 12 & 23 & 22 \\
\hline 13 & 3 & 16 & 13 & 13 & 3 & 16 & 6 & 17 & 22 & 13 & 13 & 19 & 22 & 23 \\
\hline 14 & 4 & 11 & 15 & 14 & 14 & 14 & 14 & 12 & 5 & 4 & 14 & 15 & 27 & 24 \\
\hline 15 & 5 & 12 & 14 & 15 & 15 & 15 & 15 & 15 & 15 & 5 & 5 & 14 & 29 & 25 \\
\hline 16 & 6 & 13 & 16 & 16 & 6 & 13 & 16 & 18 & 25 & 6 & 16 & 20 & 28 & 26 \\
\hline 17 & 7 & 17 & 18 & 7 & 17 & 18 & 24 & 13 & 8 & 17 & 17 & 17 & 24 & 27 \\
\hline 18 & 8 & 18 & 17 & 8 & 18 & 17 & 25 & 16 & 18 & 18 & 8 & 18 & 26 & 28 \\
\hline 19 & 9 & 19 & 19 & 9 & 9 & 20 & 26 & 19 & 28 & 19 & 19 & 13 & 25 & 29 \\
\hline 20 & 10 & 20 & 20 & 10 & 10 & 19 & 32 & 20 & 35 & 10 & 10 & 16 & 30 & 30 \\
\hline 21 & 21 & 24 & 22 & 24 & 22 & 21 & 7 & 21 & 3 & 27 & 23 & 21 & 11 & 11 \\
\hline 22 & 22 & 25 & 21 & 25 & 21 & 22 & 8 & 24 & 13 & 28 & 31 & 22 & 13 & 12 \\
\hline 23 & 23 & 26 & 23 & 26 & 31 & 26 & 9 & 27 & 23 & 29 & 21 & 29 & 12 & 13 \\
\hline 24 & 24 & 21 & 25 & 21 & 25 & 24 & 17 & 22 & 6 & 34 & 26 & 25 & 17 & 14 \\
\hline 25 & 25 & 22 & 24 & 22 & 24 & 25 & 18 & 25 & 16 & 35 & 32 & 24 & 19 & 15 \\
\hline 26 & 26 & 23 & 26 & 23 & 32 & 23 & 19 & 28 & 26 & 36 & 24 & 30 & 18 & 16 \\
\hline 27 & 27 & 27 & 28 & 34 & 28 & 28 & 27 & 23 & 9 & 21 & 29 & 27 & 14 & 17 \\
\hline 28 & 28 & 28 & 27 & 35 & 27 & 27 & 28 & 26 & 19 & 22 & 33 & 28 & 16 & 18 \\
\hline 29 & 29 & 29 & 29 & 36 & 33 & 30 & 29 & 29 & 29 & 23 & 27 & 23 & 15 & 19 \\
\hline 30 & 30 & 30 & 30 & 33 & 36 & 29 & 33 & 30 & 36 & 32 & 35 & 26 & 20 & 20 \\
\hline 31 & 31 & 32 & 31 & 32 & 23 & 32 & 10 & 34 & 31 & 33 & 22 & 33 & 31 & 31 \\
\hline 32 & 32 & 31 & 32 & 31 & 26 & 31 & 20 & 35 & 32 & 30 & 25 & 36 & 33 & 32 \\
\hline 33 & 33 & 33 & 33 & 30 & 29 & 36 & 30 & 36 & 33 & 31 & 28 & 31 & 32 & 33 \\
\hline 34 & 34 & 34 & 35 & 27 & 35 & 35 & 34 & 31 & 10 & 24 & 36 & 35 & 34 & 34 \\
\hline 35 & 35 & 35 & 34 & 28 & 34 & 34 & 35 & 32 & 20 & 25 & 30 & 34 & 36 & 35 \\
\hline 36 & 36 & 36 & 36 & 29 & 30 & 33 & 36 & 33 & 30 & 26 & 34 & 32 & 35 & 36 \\
\hline
\end{tabular}

Table 5: Modular transformations of genus 3 even spin structures 


\section{References}

[1] E. D'Hoker and D.H. Phong, "Two-Loop Superstrings I, Main Formulas", Phys. Lett. B529 (2002) 241-255; hep-th/0110247

[2] E. D'Hoker and D.H. Phong, "Two-Loop Superstrings II, The chiral Measure on Moduli Space", Nucl. Phys. B636 (2002) 3-60; hep-th/0110283.

[3] E. D'Hoker and D.H. Phong, "Two-Loop Superstrings III, Slice Independence and Absence of Ambiguities", Nucl. Phys. B636 (2002) 61-79; hep-th/0111016.

[4] E. D'Hoker and D.H. Phong, "Two-Loop Superstrings IV, The Cosmological Constant and Modular Forms", Nucl. Phys. B639 (2002) 129-181; hep-th/0111040.

[5] E. D'Hoker and D.H. Phong, "Lectures on two-loop superstrings", Hangzhou, Beijing 2002, hep-th/0211111.

[6] J.I. Igusa, "On the graded ring of theta constants", Amer. J. Math. 86 (1964) 219;

J.I. Igusa, "On Siegel modular forms of genus two", Amer. J. Math. 84 (1962) 175;

J.I. Igusa, "Modular forms and projective invariants", Amer. J. Math. 89 (1967) 817-855.

[7] J.I. Igusa, Theta Functions, Springer Verlag, 1972.

[8] K. Aoki, E. D'Hoker and D. H. Phong, "Two-loop superstrings on orbifold compactifications," Nucl. Phys. B 688 (2004) 3-69 arXiv:hep-th/0312181.

[9] E. D'Hoker and D.H. Phong, "The geometry of string perturbation theory", Rev. Modern Physics 60 (1988) 917-1065.

[10] E. D'Hoker and D.H. Phong, "Asyzygies, modular forms, and the superstring measure II", to appear in hep-th.

[11] S. Kachru, J. Kumar, and E. Silverstein, "Vacuum energy cancellation in a nonsupersymmetric string", Phys. Rev D59 (1999) 106004, hep-th/9807076.

S. Kachru and E. Silverstein, "Self-dual nonsupersymmetric Type II string compactifications", JHEP 9811:001 (1998) hep-th/9808056

S. Kachru and E. Silverstein, "On vanishing two loop cosmological constants in nonsupersymmetric strings", JHEP 9901:004 (1999) hep-th/9810129 48

G. Shiu and S. H. H. Tye, "Bose-Fermi degeneracy and duality in non-supersymmetric strings," Nucl. Phys. B 542, 45 (1999) arXiv:hep-th/9808095.

[12] E. Freitag, "Hilbert modular forms", Springer-Verlag, Berlin, 1990;

E. Freitag, "Siegelsche Modulfunktionen", Springer-Verlag, 1983. 
[13] A. Belavin, A. Polyakov, and A. Zamolodchikov, "Infinite conformal symmetry in twodimensional quantum field theory", Nuclear Phys. B 241 (1984) 333-380;

D. Friedan and S. Shenker, "The analytic geometry of two-dimensional conformal field theory", Nuclear Phys. B 281 (1987) 509-545;

D. Friedan and S. Shenker, "The integrable analytic geometry of quantum string", Phys. Lett. B 175 (1986) 287-296.

[14] J. Fay Theta Functions on Riemann surfaces, Springer Lecture Notes in Mathematics, No 352, Springer Berlin (1973). 